Research Article

\title{
Two-Step Unconditionally Stable Noniterative Dissipative Displacement Method for Analysis of Nonlinear Structural Dynamics Problems
}

\author{
Changqing Li $\mathbb{D}^{D}$, Baoyi Sheng $\mathbb{D}$, Zhipeng Lai $\mathbb{D}^{\mathbb{D}}$, Lizhong Jiang $\mathbb{D}$, and Ping Xiang $\mathbb{D}$ \\ School of Civil Engineering, Central South University, Changsha, Hunan, China \\ Correspondence should be addressed to Zhipeng Lai; laizhipeng2016@163.com
}

Received 12 December 2019; Revised 21 February 2021; Accepted 4 March 2021; Published 21 April 2021

Academic Editor: Hamid Toopchi-Nezhad

Copyright (c) 2021 Changqing Li et al. This is an open access article distributed under the Creative Commons Attribution License, which permits unrestricted use, distribution, and reproduction in any medium, provided the original work is properly cited.

\begin{abstract}
When solving structural dynamic problems, the displacement algorithm needs only calculating and storing structure's displacements in the main calculation process, which makes the displacement algorithm have advantages over multivariable algorithms in calculation efficiency and storage requirements. By using a novel approach based on dimensional analysis firstly given by the first author, a one-parameter family of two-step unconditionally stable noniterative displacement algorithms, referred to as the CQ- $2 x$ method, is developed. Compared with other unconditionally stable noniterative multivariable algorithms such as the representative KR- $\alpha$ method, the proposed method has advantages in several aspects. The CQ- $2 x$ method is unconditionally stable regardless of stiffness hardening or stiffness weakening, while the KR- $\alpha$ method is only conditionally stable in case of stiffness hardening. The CQ- $2 x$ method needs only one solver within one time step, while the KR- $\alpha$ method needs two solvers within one time step, which makes the CQ- $2 x$ method show higher efficiency. Numerical examples are presented to demonstrate the potential of the proposed method.
\end{abstract}

\section{Introduction}

Direct integration methods are commonly used to solve structural dynamics equations. According to whether iteration is needed to solve nonlinear dynamic problems, these methods can be divided into two categories: implicit and noniterative. If the solution for the next time step depends on the responses in future time steps, then the algorithm is classified as an implicit algorithm [1,2]. The well-known Houbolt [3], Newmark [4], Wislon [5], HHT- $\alpha$ [6], WBZ $\alpha$ [7], generalized- $\alpha$ [8], and Bath [9-11] algorithms are examples of implicit algorithms. When the implicit methods are used to solve nonlinear structural dynamics equations, an iterative scheme (for example, the Newton-Raphson algorithm) is required in each time step which is often complicated and time consuming.

By contrast, equilibrium in noniterative algorithms is satisfied in the previous time step rather than the current step; consequently, such algorithms do not require the formation of the tangent stiffness matrix or any iterative calculations and are computationally more efficient than implicit algorithms in the nonlinear case when using same time step size.

The central difference method (CDM) $[1,2]$ is the most common and effective noniterative method since it not only needs no nonlinear iteration but also needs no solver for nonlinear problems. However, the CDM is only conditionally stable, which means extraordinary small time step size must be used in order to make the calculation stable. The conditionally stable CDM is preferred when the time step size required for accuracy is of the same order as the stability limit of the algorithm, e.g., in wave propagation problems $[2,12]$. Nevertheless, unconditionally stable noniterative algorithms are more useful for solving nonlinear structural dynamics problems.

Chang's algorithms [13-16], the CR [17] method, and the KR- $\alpha$ [18] method are examples of unconditionally stable noniterative algorithms. The first unconditionally stable 
noniterative structure-dependent integration method was developed by Chang [13]. An assessment of such structuredependent unconditionally stable noniterative and semiexplicit (SE) algorithms for application to dynamic problems has been presented by Kolay and Ricles [19]. These authors found that an SE algorithm may generate large damping forces when a realistic time step size is used for the nonlinear transient analysis of realistic structural systems subjected to seismic excitations, whereas the KR- $\alpha$ method [18] may produce high-frequency overshoot. Furthermore, unconditionally stable noniterative model-dependent algorithms may become conditionally stable when severe stiffness hardening occurs, and most of them require the solution of linear equations whose coefficient matrix is nondiagonal twice within the one time step, which lowers their computational efficiency. When the number of DOFs of the structure is large, the majority of the computation time of an algorithm is consumed in solving linear equations whose coefficient matrices are nondiagonal. From the perspective of computational efficiency, it is preferable for an algorithm to solve a system of linear equations only once per time step.

In conjunction with the total energy framework and a generalized time-weighted residual approach, Har and Tamma [20,21] have provided good guidance for developing new time integration algorithms and procedures for evaluating new and existing algorithms. However, the unconditionally stable noniterative structure-dependent integration methods can hardly be evaluated by those guidances. The algorithms including the structure-dependent algorithm may be easier to be designed by using a novel approach developed by the first author of the current study based on dimensional analysis. Most of the commonly used algorithms for structural dynamics analysis consist of one motion equation and two difference equations of displacement and velocity. By contrast, the novel dimensional analysis approach presented here relies on only a simple objective function or functions (the case of multiple functions will not be discussed in this paper), and the assumptions concerning displacement, velocity, and acceleration are implicitly included in their Taylor series expansions and the dynamic equilibrium equation.

When solving structural dynamic problems, according to the types of physical quantities that must be involved in the main calculation steps, the algorithm can be divided into the single variable method and multivariable method. As a single variable method, the displacement method needs only calculating and storing displacements in the calculation process, which makes the displacement method have advantages over other multivariable algorithms in calculation efficiency and storage requirements. So the novel approach was applied firstly to design the displacement method. By using this novel approach, a two-step unconditionally stable noniterative displacement algorithm (NDA) called the CQ$2 x$ method (a two-step algorithm named after the first author) has been proposed [22]. The CQ-2x method combines unconditional stability with noniterative formulations of both displacement and velocity, the use of one solver per time step, and no overshoot in either displacement or velocity. However, CQ-2x does not offer controllable numerical dissipation. To introduce controllable numerical damping, while simultaneously preserving the previously mentioned desirable numerical characteristics of the CQ- $2 x$ algorithm, a three-step displacement algorithm called the CQ-3 method [23] with controllable numerical dissipation has also been developed. However, the need for three steps is a disadvantage that may lower the efficiency of the algorithm. Thus, the development of a family of two-step unconditionally stable NDAs with controllable numerical damping is the main task of this paper.

The article begins with an objective function for a twostep NDA. Then, the preliminary form of the two-step NDA is obtained through dimensional analysis. Since there are 9 coefficients to be determined in the preliminary form of the NDA, equations for determining those coefficients from the algorithm's consistency and stability are provided. Since the algorithm's consistency and stability requirements cannot be used to determine all coefficients, the remaining free coefficients $b_{5}, b_{7}$, and $b_{8}$ are then determined from other characteristics of the algorithm, such as the relative period error and numerical damping ratio. A self-starting procedure is presented, and the overshoots in displacement and velocity are analyzed; subsequently, three numerical examples are provided to demonstrate the accuracy, stability, and efficiency of the proposed method.

\section{Preliminary Form of NDA}

For a single-DOF system, the linear structural dynamics equation can be written as

$$
m a(t)+c v(t)+k u(t)=f(t) .
$$

In the equation above, $m, c$, and $k$ represent the mass, damping, and stiffness, respectively; $a, v$, and $u$ represent the acceleration, velocity, and displacement of the mass, respectively; $f$ is the external force; and $t$ is the time.

When no nonlinear damping is considered, the nonlinear structural dynamics equation can be written as follows [18]:

$$
m a(t)+c v(t)+f_{\text {in }}(u, t)=f_{\text {ext }}(t),
$$

where $f_{\text {ext }}(t)$ is the external force.

The nonlinearity of a structure may be complex. In some cases, the structural nonlinearity can be expressed in terms of nonlinear damping and nonlinear stiffness. In these cases, the nonlinear structural dynamics equation can be rewritten as

$$
m a(t)+c(t) v(t)+k(t) u(t)=f(t)
$$

where the nonlinear damping $c(t)$ and the nonlinear stiffness $k(t)$ are given by

$$
\begin{aligned}
& k(t)=k(u(t), v(t), t), \\
& c(t)=c(u(t), v(t), t) .
\end{aligned}
$$

In order to obtain the numerical solution at the discrete time point, according to the definition of the "noniterative" displacement algorithm given above, the objective of a twostep NDA is to find $u_{t+2 \Delta t}$ when $u_{t+\Delta t}, u_{t}, f_{t+\Delta t}, m, c_{t+\Delta t}$, 
$k_{t+\Delta t}$, and $\Delta t$ are given; this can be expressed by the objective function given in equation (5) where $u_{t+2 \Delta t}$ is regarded as a dependent variable and $u_{t+\Delta t}, u_{t}, f_{t+\Delta t}, m, c_{t+\Delta t}, k_{t+\Delta t}$, and $\Delta t$ are regarded as independent variables:

$$
u_{t+2 \Delta t}=u_{t+2 \Delta t}\left(m, c_{t+\Delta t}, k_{t+\Delta t}, f_{t+\Delta t}, u_{t+\Delta t}, u_{t}, \Delta t\right),
$$

where $\Delta t$ is the time step size and the subscripts denote discrete time points. Because the displacement algorithm is to be designed, there are no velocity and acceleration occurring in equation (5). A displacement algorithm designed based on dimensional analysis to solve the objective function given in equation (5) can be written as follows:

$$
\left(m+b_{1} c_{t+\Delta t} \Delta t+b_{2} k_{t+\Delta t} \Delta t^{2}\right) u_{t+2 \Delta t}=\left(b_{3} m+b_{4} c_{t+\Delta t} \Delta t+b_{5} k_{t+\Delta t} \Delta t^{2}\right) u_{t+\Delta t}
$$

where $b_{i}$ are undetermined coefficients. The detailed derivation of equation (6) based on dimensional analysis is presented in Appendix A. $b_{i}$ can be determined from the NDA's consistency and stability requirements.

\section{Consistency Analysis to Determine $b_{i}$}

The consistency of an NDA of the form given in equation (6) can be analyzed by calculating the local algorithmic error. To calculate the local algorithmic error $\varepsilon_{u_{2+3 \Delta t}}$ between the exact displacement $u(t+2 \Delta t)$ and the computed displacement $u_{t+2 \Delta t}$, it is assumed that, in equation (6),

$$
\begin{aligned}
u_{t} & =u(t), \\
u_{t+\Delta t} & =u(t+\Delta t), \\
u_{t+2 \Delta t} & =u(t+2 \Delta t)-\varepsilon_{u_{t+2 \Delta t}}, \\
c_{t+\Delta t} & =c(t+\Delta t), \\
k_{t+\Delta t} & =k(t+\Delta t), \\
f_{t+\Delta t} & =f(t+\Delta t),
\end{aligned}
$$

where a quantity with brackets "()" denotes an exact quantity and a quantity without brackets "()" but with a subscript denotes a computed quantity.

Substituting equations (7a)-(7f) into equation (6) yields the following:

$$
\begin{aligned}
\left(m+b_{1} c(t+\Delta t) \Delta t+b_{2} k(t+\Delta t) \Delta t^{2}\right) \varepsilon_{u_{t+2 \Delta t}} \\
=\left(m+b_{1} c(t+\Delta t) \Delta t+b_{2} k(t+\Delta t) \Delta t^{2}\right) u(t+2 \Delta t) \\
\quad-\left(b_{3} m+b_{4} c(t+\Delta t) \Delta t+b_{5} k(t+\Delta t) \Delta t^{2}\right) u(t+\Delta t) \\
\quad-\left(b_{6} m+b_{7} c(t+\Delta t) \Delta t+b_{8} k(t+\Delta t) \Delta t^{2}\right) u(t)-b_{9} f(t+\Delta t) \Delta t^{2} .
\end{aligned}
$$

Taylor series expansions of $u(t+\Delta t), u(t+2 \Delta t)$, $v(t+\Delta t)$, and $a(t+\Delta t)$ can be written as follows:

$$
+\left(b_{6} m+b_{7} c_{t+\Delta t} \Delta t+b_{8} k_{t+\Delta t} \Delta t^{2}\right) u_{t}+b_{9} f_{t+\Delta t} \Delta t^{2}
$$$$
u(t+\Delta t)=u(t)+v(t) \Delta t+\frac{1}{2} a(t) \Delta t^{2}+\frac{1}{6} \dot{a}(t) \Delta t^{3}+\cdots,
$$

$$
u(t+2 \Delta t)=u(t)+2 v(t) \Delta t+2 a(t) \Delta t^{2}+\frac{4}{3} \dot{a}(t) \Delta t^{3}+\cdots,
$$

$$
v(t+\Delta t)=v(t)+a(t) \Delta t+\frac{1}{2} \dot{a}(t) \Delta t^{2}+\frac{1}{6} \ddot{a}(t) \Delta t^{3}+\cdots,
$$

$$
a(t+\Delta t)=a(t)+\dot{a}(t) \Delta t+\frac{1}{2} \ddot{a}(t) \Delta t^{2}+\frac{1}{6} \stackrel{\dot{t}}{a}(t) \Delta t^{3}+\cdots,
$$

where a superscripted dot denotes differentiation with respect to time. The nonlinear dynamic equilibrium of equation (3) in step $t+\Delta t$ is written as

$$
\begin{aligned}
f(t+\Delta t)= & m a(t+\Delta t)+c(t+\Delta t) v(t+\Delta t) \\
& +k(t+\Delta t) u(t+\Delta t) .
\end{aligned}
$$

Substituting equations (9a), (9c), and (9d) into equation (10) yields

$$
\begin{aligned}
f(t+\Delta t)= & m(a(t)+\dot{a}(t) \Delta t+\cdots)+c(t+\Delta t)(v(t) \\
& +a(t) \Delta t+\cdots)+k(t+\Delta t)(u(t)+v(t) \Delta t+\cdots) .
\end{aligned}
$$

Substituting equations (9a), (9b), and (11) into equation (8) yields

$$
\begin{aligned}
& \left(m+b_{1} c(t+\Delta t) \Delta t+b_{2} k(t+\Delta t) \Delta t^{2}\right) \varepsilon_{u_{t+2 \Delta t}} \\
& =e_{0}+e_{1} \Delta t+e_{2} \Delta t^{2}+e_{3} \Delta t^{3}+e_{4} \Delta t^{4}+e_{5} \Delta t^{5} \cdots,
\end{aligned}
$$

where the expressions for $e_{0}, e_{1}, e_{2}, e_{3}, e_{4}$, and $e_{5}$ are given in Appendix B.

Rewriting equation (12) yields 


$$
\begin{aligned}
\lim _{\Delta t \longrightarrow 0} \varepsilon_{u_{t+2 \Delta t}} & =\lim _{\Delta t \longrightarrow 0} \frac{e_{0}+e_{1} \Delta t+e_{2} \Delta t^{2}+e_{3} \Delta t^{3}+e_{4} \Delta t^{4}+e_{5} \Delta t^{5} \cdots}{m+b_{1} c(t+\Delta t) \Delta t+b_{2} k(t+\Delta t) \Delta t^{2}} \\
& =\lim _{\Delta t \rightarrow 0} \frac{e_{0}+e_{1} \Delta t+e_{2} \Delta t^{2}+e_{3} \Delta t^{3}+e_{4} \Delta t^{4}+e_{5} \Delta t^{5} \cdots}{m}
\end{aligned}
$$

The statement that the NDA has a nominal $n$th order time accuracy of displacement means that the following holds:

$$
\lim _{\Delta t \rightarrow 0} \frac{\varepsilon_{u_{t+2 \Delta t}}}{\Delta t^{n}}=0
$$

The combination of equations (13) and (14) leads to

$$
\lim _{\Delta t \longrightarrow 0} \frac{\varepsilon_{u_{t+2 \Delta t}}}{\Delta t^{n}}=\frac{1}{m}\left(\frac{e_{0}}{\Delta t^{n}}+\frac{e_{1}}{\Delta t^{n-1}}+\frac{e_{2}}{\Delta t^{n-2}}+\frac{e_{3}}{\Delta t^{n-3}}+\cdots+\right)=0 .
$$

Substituting $n=2$ into equation (15) yields the condition that must be met for the NDA to achieve a nominal secondorder time accuracy of displacement:

$$
\lim _{\Delta t \longrightarrow 0} \frac{\varepsilon_{u_{t+2 \Delta t}}}{\Delta t^{2}}=\frac{1}{m}\left(\frac{e_{0}}{\Delta t^{2}}+\frac{e_{1}}{\Delta t}+e_{2}+e_{3} \Delta t+\cdots+\right)=0 .
$$

Equation (16) is satisfied only when $e_{0}=e_{1}=e_{2}=0$, and the conditions for which can be obtained by simultaneously solving equations (B.2a), (B.2b), and (B.2c) (the detailed derivation can be seen in Appendix B):

$$
\begin{aligned}
& b_{1}=1-b_{7}, \\
& b_{2}=1+b_{8}+b_{5}, \\
& b_{3}=2, \\
& b_{4}=1-2 b_{7}, \\
& b_{6}=-1, \\
& b_{9}=1 .
\end{aligned}
$$

Equation (17) ensures that the NDA will achieve a nominal second-order time accuracy of displacement.

To confirm the nominal second-order time accuracy of displacement achieved with equation (17), we can substitute equation (17) into equation (12) and obtain the following equation:

$$
\varepsilon_{u_{t+2 \Delta t}}=\frac{-\Delta t^{3}\left(120\left(1+b_{5}+2 b_{8}\right) \omega_{t+\Delta t}^{2} v_{t}+120\left(1-2 b_{7}\right) a_{t} \xi_{t+\Delta t} \omega_{t+\Delta t}\right)+9 \Delta t^{4}}{120\left(b_{5}+b_{8}+1\right) \Omega_{t+\Delta t}^{2}+240\left(1-b_{7}\right) \xi_{t+\Delta t} \Omega_{t+\Delta t}+120},
$$

where

$$
\begin{aligned}
\omega_{t+\Delta t} & =\sqrt{\frac{k(t+\Delta t)}{m}}, \\
\xi_{t+\Delta t} & =\frac{c(t+\Delta t)}{2 m \omega_{t+\Delta t}}, \\
\Omega_{t+\Delta t} & =\omega_{t+\Delta t} \Delta t .
\end{aligned}
$$

The substitution of equation (17) into equation (6) yields an NDA with a nominal second-order time accuracy of displacement:

$$
\begin{aligned}
& \left(m+\left(1-b_{7}\right) c_{t+\Delta t} \Delta t+\left(1+b_{8}+b_{5}\right) k_{t+\Delta t} \Delta t^{2}\right) u_{t+2 \Delta t} \\
= & \left(2 m+\left(1-2 b_{7}\right) c_{t+\Delta t} \Delta t+b_{5} k_{t+\Delta t} \Delta t^{2}\right) u_{t+\Delta t} \\
& +\left(-m+b_{7} c_{t+\Delta t} \Delta t+b_{8} k_{t+\Delta t} \Delta t^{2}\right) u_{t}+f_{t+\Delta t} \Delta t^{2} .
\end{aligned}
$$

It can be seen from the derivation of equation (20) that the NDA is derived from only one objective function, equation (5). The possible assumed relationships among the displacement, velocity, and acceleration are all implied in the Taylor series expansions (as seen in equations (9a)-(9d)) and the nonlinear dynamic equilibrium equation (as seen in equation (10)). Newmark's assumptions (with either constant coefficients or structure-dependent coefficients) concerning displacement and velocity are not needed for the NDA family derived in this paper although they are needed for most other algorithms; thus, this derivation is more comprehensive and allows no promising algorithms to be missed when the objective function is determined. It should be noted here that the NDA accuracy analysis applies to the case of nonlinear structural dynamics since nonlinear equation (10) is used in the derivation instead of linear equation (1), which is also a point of difference with respect to other algorithms.

When calculation of the velocity is necessary, it can be achieved with acceptable accuracy by means of any applicable displacement difference approximation. For example, one can use, but is not limited to, the displacement difference approximation used in the central-eccentric difference method [24]:

$$
v_{t+2 \Delta t}=\frac{3 u_{t+2 \Delta t}-4 u_{t+\Delta t}+u_{t}}{2 \Delta t} .
$$

Equation (21) has a nominal time accuracy of velocity of at least first order, and the local algorithmic velocity error 
$\varepsilon_{v_{t+2 \Delta t}}\left(=v(t+2 \Delta t)-v_{t+2 \Delta t}\right)$ when this approximation is applied in the proposed CQ- $2 x$ method is

$$
\varepsilon_{v_{t+2 \Delta t}}=\frac{-\Delta t^{2}\left(80 \dot{a}_{t}+360\left(1+b_{5}+2 b_{8}\right) \omega_{t+\Delta t}^{2} v_{t}+360\left(1-2 b_{7}\right) \xi_{t+\Delta t} \omega_{t+\Delta t} a_{t}\right)+\varphi\left(\Delta t^{3}\right)}{240\left(b_{5}+b_{8}+\right) \Omega^{2}+480\left(1-b_{7}\right) \xi \Omega+240} .
$$

After the noniterative calculation of $u_{t+2 \Delta t}$ and $v_{t+2 \Delta t}$, $a_{t+2 \Delta t}$ can be calculated from the nonlinear dynamic equilibrium (as given in equation (3)) at step $t+2 \Delta t$ :

$$
a_{t+2 \Delta t}=\frac{1}{m}\left(f_{t+2 \Delta t}-c_{t+2 \Delta t} v_{t+2 \Delta t}-k_{t+2 \Delta t} u_{t+2 \Delta t}\right) .
$$

Equation (23) has a nominal time accuracy of acceleration of at least first order, and when this approximation is applied in the CQ- $2 x$ method, the local algorithmic acceleration error $\varepsilon_{a_{t+2 \Delta t}}\left(=a(t+2 \Delta t)-a_{t+2 \Delta t}\right)$ can be written as

$$
\varepsilon_{a_{t+2 \Delta t}}=\frac{\Delta t^{2} \xi_{t+2 \Delta t} \omega_{t+2 \Delta t}\left(2 \dot{a}_{t}+9\left(1+b_{5}+2 b_{8}\right) \omega_{t+\Delta t}^{2} v_{t}+9\left(1-2 b_{7}\right) \xi_{t+\Delta t} \omega_{t+\Delta t} a_{t}\right)+9\left(\Delta t^{3}\right)}{3\left(1+b_{5}+b_{8}\right) \Omega_{t+\Delta t}^{2}+6\left(1-b_{7}\right) \xi_{t+\Delta t} \Omega_{t+\Delta t}+3},
$$

where

$$
\begin{aligned}
& \omega_{t+2 \Delta t}=\sqrt{\frac{k(t+2 \Delta t)}{m}}, \\
& \xi_{t+2 \Delta t}=\frac{c(t+2 \Delta t)}{2 m \omega_{t+2 \Delta t}} .
\end{aligned}
$$

The accuracy expressed above is called the nominal accuracy because the derivation of the displacement consistency assumes that the values of $u$ at time steps $t$ and $t+$ $\Delta t$ are known exactly, which cannot be ensured, meaning that this assumption leads to additional errors. However, it can be concluded that an NDA described by equations (20), (21), and (23) has a global time accuracy of at least first order, as will be validated by Example 1 .

\section{Stability Analysis to Determine $b_{i}$}

An algorithm is convergent if and only if it is consistent and stable. The consistency of the proposed NDA family has been analyzed above. In this section, the stability of an NDA with the form given in equation (20) will be studied. It should again be noted here that the NDA consistency analysis applies to the solution of nonlinear structural dynamics problems of the form given in equation (3) because equation (3), not equation (1), is used throughout the entire derivation. For the same reason, the following NDA stability analysis also applies to the solution of nonlinear structural dynamics problems of the form given in equation (3). The NDA expressed in equation (20) can be rewritten as follows:

$$
\left\{\begin{array}{c}
u_{t+2 \Delta t} \\
u_{t+\Delta t}
\end{array}\right\}=A\left\{\begin{array}{c}
u_{t+\Delta t} \\
u_{t}
\end{array}\right\}+\left\{\begin{array}{c}
\frac{b_{9} f_{t+\Delta t} \Delta t^{2}}{m+\left(1-b_{7}\right) c_{t+\Delta t} \Delta t+\left(1+b_{8}+b_{5}\right) k_{t+\Delta t} \Delta t^{2}} \\
0
\end{array}\right\}
$$

where the amplification matrix $A$ is

$$
\begin{gathered}
A=\left[\begin{array}{cc}
A_{11} & A_{12} \\
1 & 0
\end{array}\right], \\
\% A_{11}=\frac{2 m+\left(1-2 b_{7}\right) c_{t+\Delta t} \Delta t+b_{5} k_{t+\Delta t} \Delta t^{2}}{m+\left(1-b_{7}\right) c_{t+\Delta t} \Delta t+\left(1+b_{8}+b_{5}\right) k_{t+\Delta t} \Delta t^{2}},
\end{gathered}
$$

$$
A_{12}=\frac{-m+b_{7} c_{t+\Delta t} \Delta t+b_{8} k_{t+\Delta t} \Delta t^{2}}{m+\left(1-b_{7}\right) c_{t+\Delta t} \Delta t+\left(1+b_{8}+b_{5}\right) k_{t+\Delta t} \Delta t^{2}}
$$

A stability study similar to the one conducted by Hilber and Hughes [25] is performed (the detailed derivation can be seen in Appendix C). The stability conditions for a first-order time-accurate NDA can be written as follows: 


$$
\begin{aligned}
-1 & \leq A_{2} \leq 1, \\
-\frac{1}{2}\left(1+A_{2}\right) & \leq A_{1} \leq \frac{1}{2}\left(1+A_{2}\right),
\end{aligned}
$$

where

$$
\begin{aligned}
& A_{1}=\frac{1}{2} \operatorname{tr}(A)=\frac{A_{11}+A_{22}}{2}=\frac{1}{2} \frac{2 m+\left(1-2 b_{7}\right) c_{t+\Delta t} \Delta t+b_{5} k_{t+\Delta t} \Delta t^{2}}{m+\left(1-b_{7}\right) c_{t+\Delta t} \Delta t+\left(1+b_{8}+b_{5}\right) k_{t+\Delta t} \Delta t^{2}}, \\
& A_{2}=|A|=-\frac{-m+b_{7} c_{t+\Delta t} \Delta t+b_{8} k_{t+\Delta t} \Delta t^{2}}{m+\left(1-b_{7}\right) c_{t+\Delta t} \Delta t+\left(1+b_{8}+b_{5}\right) k_{t+\Delta t} \Delta t^{2}} .
\end{aligned}
$$

By considering $\omega_{t+\Delta t}=\sqrt{k_{t+\Delta t} m^{-1}}, c_{t+\Delta t}=2 m \omega_{t+\Delta t} \xi_{t+\Delta t}$, and $\omega_{t+\Delta t} \Delta t=\Omega_{t+\Delta t}$, where $\omega_{t+\Delta t}$ is the natural frequency and $\xi_{t+\Delta t}$ is the damping ratio; equations (30a) and (30b) at time $t+\Delta t$ can be rewritten as follows:

$$
\begin{aligned}
& A_{1}=\frac{1}{2} \operatorname{tr}(A)=\frac{A_{11}+A_{22}}{2}=\frac{1}{2} \frac{2+2\left(1-2 b_{7}\right) \xi_{t+\Delta t} \Omega_{t+\Delta t}+b_{5} \Omega_{t+\Delta t}^{2}}{1+2\left(1-b_{7}\right) \xi_{t+\Delta t} \Omega_{t+\Delta t}+\left(1+b_{8}+b_{5}\right) \Omega_{t+\Delta t}^{2}} . \\
& A_{2}=|A|=-\frac{-1+2 b_{7} \xi_{t+\Delta t} \Omega_{t+\Delta t}+b_{8} \Omega_{t+\Delta t}^{2}}{1+2\left(1-b_{7}\right) \xi_{t+\Delta t} \Omega_{t+\Delta t}+\left(1+b_{8}+b_{5}\right) \Omega_{t+\Delta t}^{2}} .
\end{aligned}
$$

Substituting equations (31a) and (31b) into equations (29a) and (29b) yields the following:

$$
\begin{array}{r}
1+\left(1-b_{7}\right) 2 \xi_{t+\Delta t} \Omega_{t+\Delta t}+\left(1+b_{8}+b_{5}\right) \Omega_{t+\Delta t}^{2} \geq 0, \\
4+\left(2-4 b_{7}\right) 2 \xi_{t+\Delta t} \Omega_{t+\Delta t}+\left(1+2 b_{5}\right) \Omega_{t+\Delta t}^{2} \geq 0, \\
2 \xi_{t+\Delta t} \Omega_{t+\Delta t}+\left(1+2 b_{8}+b_{5}\right) \Omega_{t+\Delta t}^{2} \geq 0 .
\end{array}
$$

Equations (32a)-(32c) give the stability conditions for the NDA that apply when solving a nonlinear structural dynamics problem of the form given in equation (3) with at least first-order time accuracy. Equation (20) shows that the $\mathrm{CDM}$ is only one specific kind of NDA with $b_{5}=-1$, $b_{7}=1 / 2$, and $b_{8}=0$. To check the correctness of the NDA stability conditions given in equations $(32 \mathrm{a})-(32 \mathrm{c})$, one can solve for the domain of stability of the CDM simply by substituting $b_{5}=-1, b_{7}=1 / 2$, and $b_{8}=0$ into equations (32a)-(32c). Then, equations (32a)-(32c) take the following form:

$$
\begin{aligned}
1+\xi_{t+\Delta t} \Omega_{t+\Delta t} & \geq 0, \\
4-\Omega_{t+\Delta t}^{2} & \geq 0, \\
2 \xi_{t+\Delta t} \Omega_{t+\Delta t} & \geq 0 .
\end{aligned}
$$

It can be seen that equations (33a)-(33c) yield the correct stability domain for the CDM, $\Omega_{t+\Delta t}^{2} \leq 4$. Since the stiffness ratio $\delta$ (where $\delta<1$ indicates stiffness softening and $\delta>1$ indicates stiffness hardening) does not appear in the amplification matrix $A$ of CQ-2x but does appear in the amplification matrices of other algorithms (for example, Chang's algorithms [16]), which causes them to become unstable when stiffness hardening occurs, it may be concluded that when used to solve the nonlinear structural dynamics equation given in equation (3), the CQ- $2 x$ method is unconditionally stable regardless of whether stiffness hardening or softening occurs as long as equations (32a)-(32c) are always satisfied when $\Omega \in[0, \infty)$ and $\xi \in[0, \infty)$, which will be validated by Example 2 .

\section{CQ-2x Method with Controllable Numerical Dissipation}

Up to this point, three coefficients have remained undetermined: $b_{5}, b_{7}$, and $b_{8}$. These coefficients can be determined from the algorithm's characteristics of unconditional stability and acceptable numerical dispersion and dissipation.

5.1. Determination of $b_{7}$. The unconditional stability of an NDA of the form given in equation (20) means that when $\Omega \in[0,+\infty)$ and $\xi \in[0,+\infty)$, equations (32a)-(32c) are always satisfied, which requires that the following must hold:

$$
\begin{aligned}
1-b_{7} & \geq 0, \\
1+b_{8}+b_{5} & \geq 0, \\
2-4 b_{7} & \geq 0, \\
1+2 b_{5} & \geq 0, \\
1+2 b_{8}+b_{5} & \geq 0 .
\end{aligned}
$$


An intermediate variable $x$ is introduced:

$$
x=1+2 b_{8}+b_{5} .
$$

Consequently, $b_{8}$ can be written as

$$
b_{8}=\frac{1}{2}\left(x-b_{5}-1\right) \text {. }
$$

Then, simplifying equation (34) leads to

$$
\begin{aligned}
& b_{7} \leq 0.5, \\
& b_{8} \leq x, \\
& b_{5} \geq-0.5, \\
& x \geq 0 .
\end{aligned}
$$

An analysis shows that, for a damping structure, a larger $b_{7}$ leads to more satisfactory algorithmic characteristics; thus, $b_{7}$ is assigned its largest possible value of 0.5 according to equation (37):

$$
b_{7}=0.5 \text {. }
$$

Substituting equations (36) and (38) into equation (20) yields the preliminary algorithmic form of the CQ- $2 x$ method:

$$
\begin{gathered}
\left(m+0.5 c_{t+\Delta t} \Delta t+\frac{1}{2}\left(x+b_{5}+1\right) k_{t+\Delta t} \Delta t^{2}\right) u_{t+2 \Delta t}=\left(2 m+b_{5} k_{t+\Delta t} \Delta t^{2}\right) u_{t+\Delta t} \\
+\left(-m+0.5 c_{t+\Delta t} \Delta t+\frac{1}{2}\left(x-b_{5}-1\right) k_{t+\Delta t} \Delta t^{2}\right) u_{t}+f_{t+\Delta t} \Delta t^{2}
\end{gathered}
$$

It can be seen from the above analysis that there are only two coefficients, $b_{5}$ and $x$, that are undetermined in the preliminary form of the CQ- $2 x$ method. These coefficients will be determined by introducing controllable numerical dissipation into CQ- $2 x$.

5.2. Determination of $b_{5}$ and $x$. When $\xi=0$, according to equation (36), equations (31a) and (31b) can be written as

$$
\begin{aligned}
\% A_{1} & =\frac{A_{11}+A_{22}}{2}=\frac{1+(1 / 2) b_{5} \Omega^{2}}{1+\left((1 / 2) b_{5}+(1 / 2) x+(1 / 2)\right) \Omega^{2}} \\
A_{2} & =\frac{1+\left((1 / 2)+(1 / 2) b_{5}-(1 / 2) x\right) \Omega^{2}}{1+\left((1 / 2)+(1 / 2) b_{5}+(1 / 2) x\right) \Omega^{2}}
\end{aligned}
$$

The effects of $b_{5}$ and $x$ on the relative period error of the preliminary CQ-2 $x$ method have been analyzed. The results when $\Delta t / T=0.1, \quad b_{5}=-0.5,-0.3,0,0.3,1,2$, and 4 , and $x \in[0,4]$ are plotted in Figure 2 . It can be seen that, as $b_{5}$ and $x$ increase, the relative period error also increases. Thus, to achieve the best accuracy, $x$ and $b_{5}$ should be assigned their lowest possible values of 0 and -0.5 , respectively. The analysis shows that when $x=0$ and $b_{5}=-0.5$, the preliminary CQ- $2 x$ method has a nominal third-order time accuracy of displacement and a global second-order time accuracy; this will be validated by Example 1. However, if numerical dissipation is needed to filter out spurious vibrations in high-vibration modes, neither $x=0$ nor $b_{5}=$ -0.5 is appropriate.

It can be proven that when $x=0$, the numerical damping ratio is also $\bar{\xi}=0$, as shown in the following equation:

$$
\begin{aligned}
x & =0, \\
A_{2} & \geq A_{1}^{2}, \\
A_{2} & =\frac{1+\left((1 / 2)+(1 / 2) b_{5}\right) \Omega^{2}}{1+\left((1 / 2)+(1 / 2) b_{5}\right) \Omega^{2}}=1, \\
\rho(A) & =\sqrt{A_{2}}=1, \\
\bar{\xi} & =0 .
\end{aligned}
$$

It can also be proven that when $b_{5}=-0.5$, the numerical damping ratio $\bar{\xi}$ of very high vibration modes $(\Omega \longrightarrow \infty)$ is also zero, as shown in the following equation:

$$
\begin{aligned}
b_{5} & =-0.5, \\
A_{2} \leq A_{1}^{2}, & \\
\lim _{\Omega \longrightarrow \infty} \rho(A) & =\lim _{\Omega \longrightarrow \infty}\left|A_{1}-\sqrt{A_{1}^{2}-A_{2}}\right|=\left|\frac{-(1 / 4)-(1 / 2) x}{(1 / 2) x+(1 / 4)}\right|=1, \\
\lim _{\Omega \longrightarrow \infty} \bar{\xi} & =0 .
\end{aligned}
$$

To introduce an appropriate level of numerical dissipation, $b_{5}$ and $x$ must be greater than their lowest values of 0 and -0.5 , respectively. The numerical damping ratio depends on the spectral radius of the amplification matrix. It may be preferable for the spectral radius of the amplification matrix $A$ of the preliminary CQ-2x method to decrease with increasing $\Omega$ with no bifurcation. According to equations (D1a) and (D1b), no bifurcation occurs when $A_{2} \geq A_{1}^{2}$, which requires the following: 


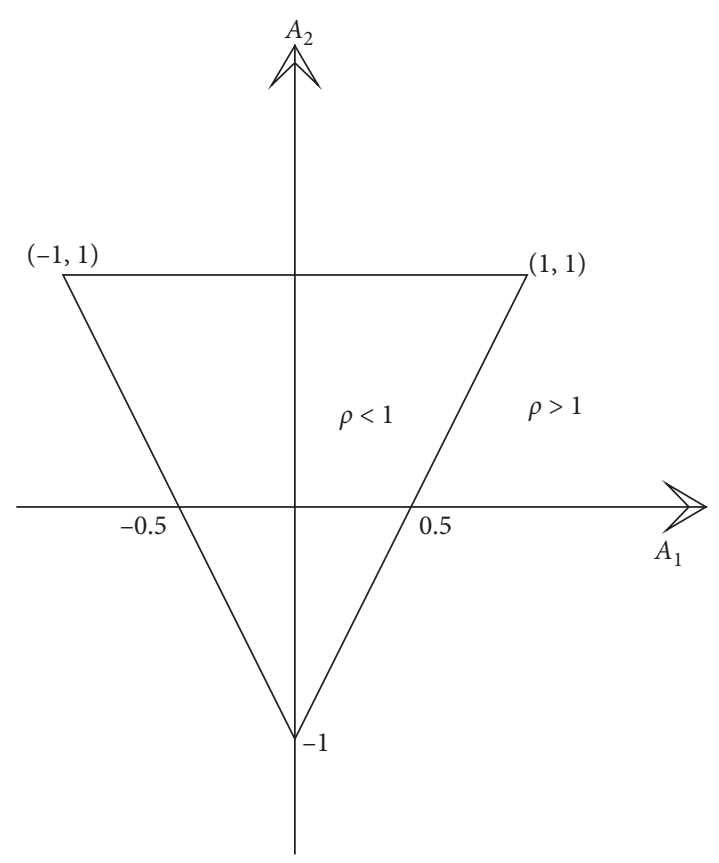

FIgURe 1: NDA stability domain.

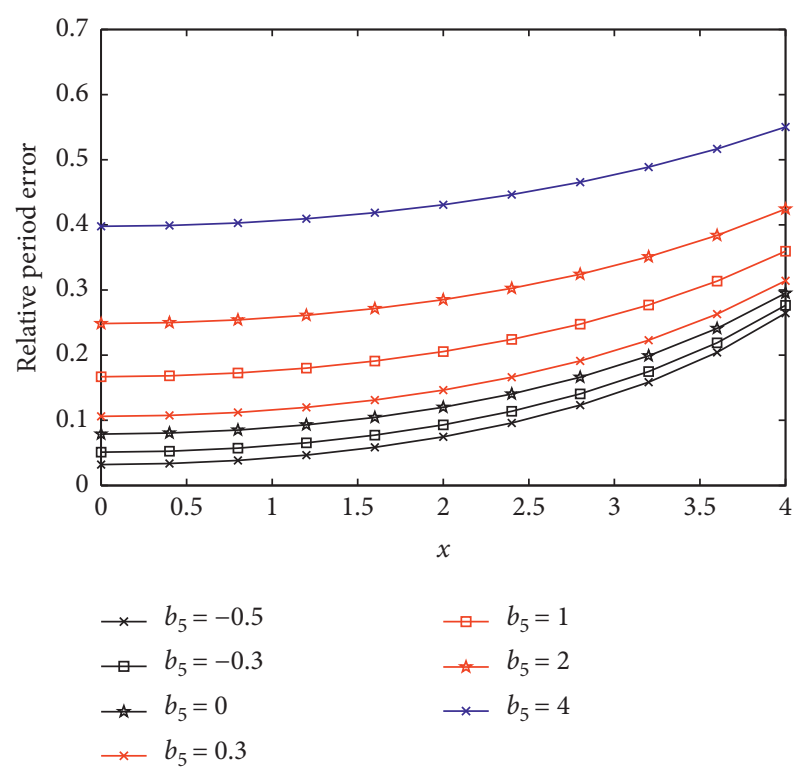

FIGURE 2: Variations in the relative period error of the preliminary CQ- $2 x$ method with $b_{5}$ and $x$ when $\Delta t / T=0.1$

$$
A_{2}-A_{1}^{2}=-\left(\frac{x^{2}}{4}-\frac{b_{5}}{2}-\frac{1}{4}\right) \Omega^{4}+\Omega^{2} \geq 0, \quad(\text { when } \Omega \in[0,+\infty))
$$

Equation (43) is satisfied when

$$
b_{5} \geq \frac{x^{2}}{2}-\frac{1}{2}
$$

$$
b_{5}=\frac{x^{2}}{2}-\frac{1}{2}
$$

Since a lower value of $b_{5}$ results in a higher accuracy, as mentioned before, $b_{5}$ is assigned the lowest possible value according to equation (44):

$$
\begin{aligned}
\left(m+\frac{1}{2} c_{t+\Delta t} \Delta t+\frac{(x+1)^{2}}{4} k_{t+\Delta t} \Delta t^{2}\right) u_{t+2 \Delta t}= & \left(2 m+\frac{x^{2}-1}{2} k_{t+\Delta t} \Delta t^{2}\right) u_{t+\Delta t} \\
& +\left(-m+\frac{1}{2} c_{t+\Delta t} \Delta t-\frac{(x-1)^{2}}{4} k_{t+\Delta t} \Delta t^{2}\right) u_{t}+f_{t+\Delta t} \Delta t^{2}
\end{aligned}
$$

Equation (46) is the final algorithmic form of displacement calculation in the CQ- $2 x$ method.

To determine $x$, while simultaneously confirming the unconditional stability of the CQ- $2 x$ method, consider the following two extreme cases: $\Omega \longrightarrow 0$ and $\Omega \longrightarrow \infty$. In accordance with equations (31a) and (31b), (36), (45), and (46) and considering the limits, it can be shown that equation $(\mathrm{C} 1)$ reduces to the following:

$$
\begin{aligned}
(\lambda-1)^{2} & =0, \quad \text { when } \Omega \longrightarrow 0, \\
\left(\lambda-\frac{x-1}{x+1}\right)^{2} & =0, \quad \text { when } \Omega \longrightarrow \infty .
\end{aligned}
$$

It can be calculated from equation (47b) that

$$
x=\frac{1-\rho_{\infty}}{1+\rho_{\infty}} .
$$

Figures 3 and 4 show how the spectral radius of the CQ$2 x$ method varies with $\rho_{\infty}$ and $\Omega$; the findings are fully consistent with the above analysis.

5.3. The Proposed Algorithm. Now that all coefficients in the preliminary NDA form presented in equation (6) have been determined, a family of unconditionally stable noniterative algorithms, collectively referred to as the CQ-2x method 


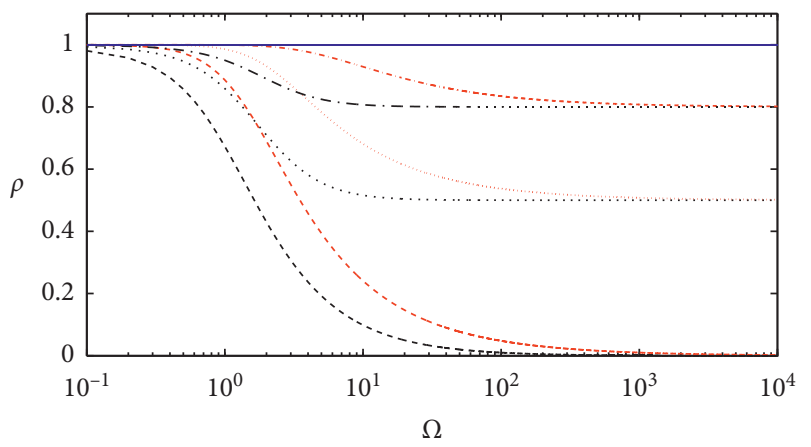

$$
\begin{aligned}
& \text { CQ- } 2 x\left(\rho_{\infty}=1\right) \\
& \ldots \text { CQ } 2 x\left(\rho_{\infty}=0.8\right) \\
& \ldots \ldots \operatorname{CQ}-2 x\left(\rho_{\infty}=0.5\right) \\
& --\operatorname{CQ}-2 x\left(\rho_{\infty}=0\right) \\
& -\operatorname{KR}\left(\rho_{\infty}=1\right)
\end{aligned}
$$

FIGURE 3: Spectral radius of CQ-2x when $\xi=0$.

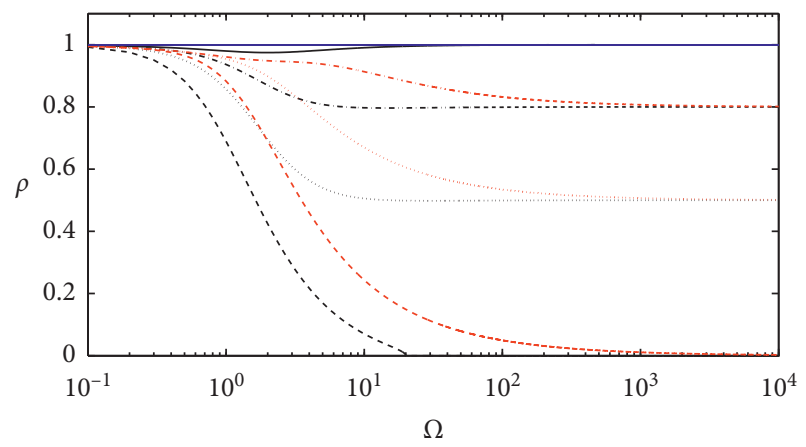

$$
\begin{aligned}
& -\operatorname{CQ}-2 x\left(\rho_{\infty}=1\right) \quad \cdots-\operatorname{KR}\left(\rho_{\infty}=0.8\right) \\
& \text {.. CQ }-2 x\left(\rho_{\infty}=0.8\right) \quad \cdots \cdots \mathrm{KR}\left(\rho_{\infty}=0.5\right) \\
& \text { … CQ }-2 x\left(\rho_{\infty}=0.5\right) \quad--\operatorname{KR}\left(\rho_{\infty}=0\right) \\
& \text {-- CQ- } 2 x\left(\rho_{\infty}=0\right) \quad \text { CAA } \\
& -\operatorname{KR}\left(\rho_{\infty}=1\right)
\end{aligned}
$$

Figure 4: Spectral radius of CQ-2x when $\xi=0.05$.

with one-parameter $x$, can be obtained by combining equations (21), (23), (46), and (48), which are rewritten as

$$
\begin{aligned}
\left(m+\frac{1}{2} c_{t+\Delta t} \Delta t+\frac{(x+1)^{2}}{4} k_{t+\Delta t} \Delta t^{2}\right) u_{t+2 \Delta t}= & \left(2 m+\frac{x^{2}-1}{2} k_{t+\Delta t} \Delta t^{2}\right) u_{t+\Delta t} \\
& +\left(-m+\frac{1}{2} c_{t+\Delta t} \Delta t-\frac{(x-1)^{2}}{4} k_{t+\Delta t} \Delta t^{2}\right) u_{t}+f_{t+\Delta t} \Delta t^{2}, \\
v_{t+2 \Delta t}= & \frac{3 u_{t+2 \Delta t}-4 u_{t+\Delta t}+u_{t}}{2 \Delta t}, \\
a_{t+2 \Delta t}= & \frac{1}{m}\left(f_{t+2 \Delta t}-c_{t+2 \Delta t} v_{t+2 \Delta t}-k_{t+2 \Delta t} u_{t+2 \Delta t}\right)
\end{aligned}
$$

where

$$
x=\frac{1-\rho_{\infty}}{1+\rho_{\infty}} .
$$

5.4. Stability for Systems with Nonlinear Stiffness. It has been demonstrated that the CQ- $2 x$ method is unconditionally stable when it is used to solve the nonlinear structural dynamics equation given in equation (3). Here, the stability of CQ-2x when it is used to solve the nonlinear structural dynamics equation given in equation (2) will be analyzed. When a general nonlinear structural dynamics problem expressed in the form of equation (2) is to be solved, equation (2) is often written in the incremental form, and the $z$ transform [17] can be used to explore an algorithm's stability for the cases of stiffness hardening and softening.
The solution to equation (2) is also often written in the incremental form, as in the following equation:

$m\left(a_{t+\Delta t}-a_{t}\right)+c\left(v_{t+\Delta t}-v_{t}\right)+k_{\tan }\left(u_{t+\Delta t}-u_{t}\right)=f_{t+\Delta t}-f_{t}$,

where $k_{\tan }$ is the tangent stiffness at time step $t+\Delta t[18]$.

When addressing structural dynamic nonlinearity in the form of equation (2), the CQ-2x method uses equations (46), (21), and (50).

The stability of an integration algorithm is generally analyzed using one of two approaches, namely, recurrence relations using an amplification matrix or discrete control theory. The unconditional stability of CQ- $2 x$ when solving nonlinear equation (3) has been analyzed through the use of an amplification matrix. In this section, discrete control 
theory will be used to analyze the stability of CQ- $2 x$ when solving nonlinear equation (2). The discrete $z$ transform and its real translation property are defined as follows [18]:

$$
\begin{aligned}
X(z) & =Z\{x[n]\}=\sum_{n=0}^{\infty} x[n] z^{-n}, \\
Z\{x[n-k]\} & =z^{-k} X(z), \\
Z\left(u_{(i+1) \Delta t}\right) & =X(z), \\
Z\left(v_{(i+1) \Delta t}\right) & =X_{v}(z), \\
Z\left(a_{(i+1) \Delta t}\right) & =X_{a}(z), \\
Z\left(f_{(i+1) \Delta t}\right) & =F(z) .
\end{aligned}
$$

The stability of CQ-2x when solving nonlinear equation (2) can be analyzed by applying discrete control theory to equations (46), (21), and (50).

First, the stability analysis of equation (46) using the $z$ transform is considered. By applying the $z$ transform and its real translation property to equation (46), this equation can be solved to determine $X(z)$ in terms of the system properties, the time step, and $F(z)$. This solution leads to the discrete transfer function of the algorithm, which is defined as the ratio of the $z$ transforms of the output $u_{i+1}$ and the input $f_{i+1}$ and can be expressed in the following general form:

$$
\frac{X(z)}{F(z)}=\frac{4 z}{d_{2} z^{2}+d_{1} z+d_{0}},
$$

where

$$
\begin{aligned}
& d_{2}=m\left[\left(x^{2}+2 x+1\right) \Omega_{t+\Delta t}^{2}+4 \xi_{t+\Delta t} \Omega_{t+\Delta t}+4\right], \\
& d_{1}=m\left[\left(2-2 x^{2}\right) \Omega_{t+\Delta t}^{2}-8\right], \\
& d_{0}=m\left[\left(x^{2}-2 x+1\right) \Omega_{t+\Delta t}^{2}-4 \xi_{t+\Delta t} \Omega_{t+\Delta t}+4\right] .
\end{aligned}
$$

It can be seen that the denominator of the transfer function in equation (52) has the same form as the characteristic equation given in equation (C1) for the amplification matrix of equation (46), whose unconditional stability has been proven.

Since the displacement calculation is unconditionally stable, the velocity calculation in equation (21), which depends solely on the displacement, is also naturally unconditionally stable.

Now, the stability of equation (50) will be analyzed. By combining equations (46), (21), and (50), the transfer function defined as the ratio of the $z$ transforms of the output $a_{i+1}$ and the input $f_{i+1}$ can be expressed in the following general form:

$$
\frac{X_{a}(z)}{F(z)}=\frac{n_{3} z^{3}+n_{2} z^{2}+n_{1} z+n_{0}}{d_{3} z^{3}+d_{2} z^{2}+d_{1} z},
$$

where

$$
\begin{aligned}
& d_{3}=m \Delta t\left[\left(x^{2}+2 x+1\right) \Omega_{t+\Delta t}^{2}+4 \xi_{t+\Delta t} \Omega_{t+\Delta t}+4\right], \\
& d_{2}=m \Delta t\left[\left(2-2 x^{2}\right) \Omega_{t+\Delta t}^{2}-8\right] \\
& d_{1}=m \Delta t\left[\left(x^{2}-2 x+1\right) \Omega_{t+\Delta t}^{2}-4 \xi_{t+\Delta t} \Omega_{t+\Delta t}+4\right] .
\end{aligned}
$$

Since $n_{3}, n_{2}, n_{1}$, and $n_{0}$ do not affect the algorithm's stability, for brevity, they are not listed in this paper.

Although the denominators of equations (52) and (54) differ by a multiplicative factor $\Delta t$, their representative characteristic equations are the same, which means that the acceleration calculation using equation (50) is also unconditionally stable. An analysis shows that the ratio of $k_{\tan }$ to $k_{t+\Delta t}$ only affects the value of the numerator of equation (54). Thus, it can be concluded that CQ-2x is unconditionally stable when it is used to solve the structural nonlinear dynamics equation given in equation (2); this will be validated by Example 2.

5.5. Accuracy Analysis for the Linear System. The accuracy of an integration algorithm generally depends on its numerical dispersion and energy dissipation characteristics. For the purposes of an accuracy analysis, the solutions to a free vibration problem obtained using CQ- $2 x$ can be written in the form of equation (D3). The two principal roots (no spurious real root) can be used to analyze the numerical dispersion and energy dissipation characteristics. The first characteristic is generally expressed in terms of the relative period error, as defined in equation (D6). The latter one can be expressed in terms of the numerical damping ratio $\bar{\xi}$, as defined in equation (D4).

Figures 5 and 6 show the relative period errors $(\mathrm{Pe})$ and the equivalent damping ratios $(\bar{\xi})$, respectively, of the proposed CQ- $2 x$ method and the KR- $\alpha$ method for various values of $\rho_{\infty}$. As shown in the figures, both $\mathrm{Pe}$ and $\bar{\xi}$ increase with decreasing $\rho_{\infty}$, and $\rho_{\infty}=0$ produces the maximum Pe and $\bar{\xi}$.

Figure 5 shows that the numerical dispersion of CQ- $2 x$ is similar to that of the KR- $\alpha$ method for the same value of $\rho_{\infty}$ when $\rho_{\infty} \neq 1$. Figure 6 shows that the KR- $\alpha$ method's energy dissipation is better than that of CQ-2x. However, at small values of $\Omega$, both $\mathrm{Pe}$ and $\bar{\xi}$ are small for CQ- $2 x$ $\left(\rho_{\infty} \in[0.8,1]\right)$ regardless of the value of $\rho_{\infty}$, which means that the lower-mode responses in an MDOF system are negligibly affected by the numerical damping. By contrast, $\bar{\xi}$ increases with increasing $\Omega$, indicating that the undesired higher modes can be effectively damped out. Note that, for $\rho_{\infty}=1$, the CQ- $2 x$ method exhibits no numerical energy dissipation, and the Pe value becomes identical to that of the KR- $\alpha\left(\rho_{\infty}=1\right)$ method and Newmark's CAA method.

5.6. Self-Starting and Overshoot. There are several possible methods of self-starting, which is a term that refers to a parameterless unified starting procedure [26]. In this paper, 


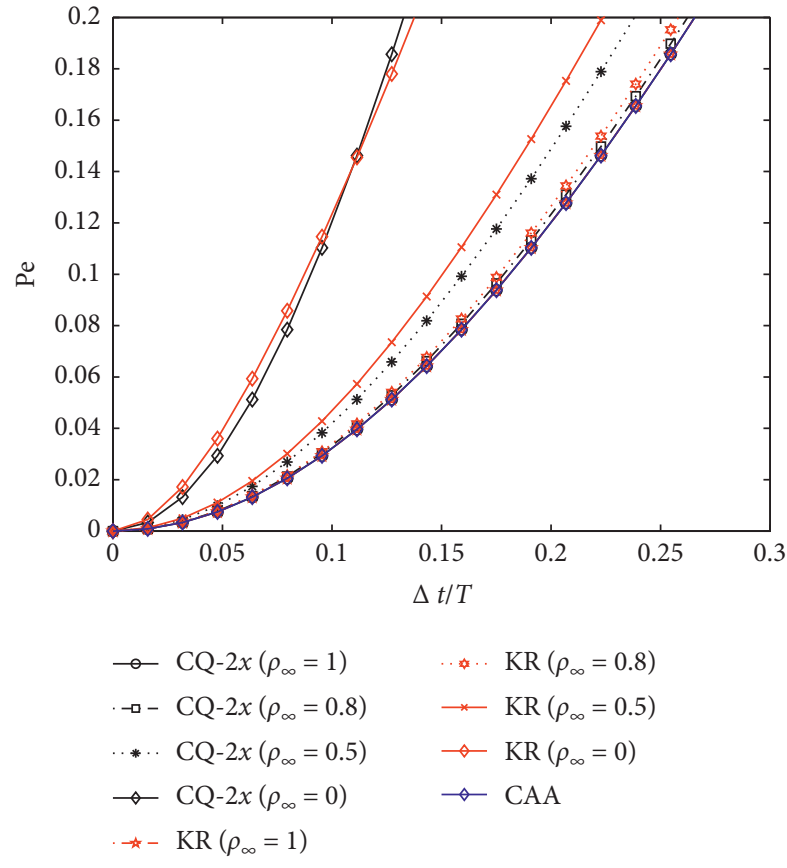

FIgURE 5: Relative period error of CQ- $2 x$ when $\xi=0$.

the self-starting of CQ- $2 x$ relies on $u_{-\Delta t}$, as calculated using the CDM, as shown in the following equation:

$$
u_{-\Delta t}=u_{0}-v \Delta t+\frac{1}{2} a_{0} \Delta t^{2}
$$

When $t=-\Delta t$, equation (46) can be written as

$$
\begin{gathered}
\left(m+\frac{1}{2} c \Delta t+\frac{(x+1)^{2}}{4} k_{0} \Delta t^{2}\right) u_{\Delta t}=\left(2 m+\frac{x^{2}-1}{2} k_{0} \Delta t^{2}\right) u_{0} \\
+\left(-m+\frac{1}{2} c \Delta t-\frac{(x-1)^{2}}{4} k_{0} \Delta t^{2}\right) u_{-\Delta t}+f_{0} \Delta t^{2} .
\end{gathered}
$$

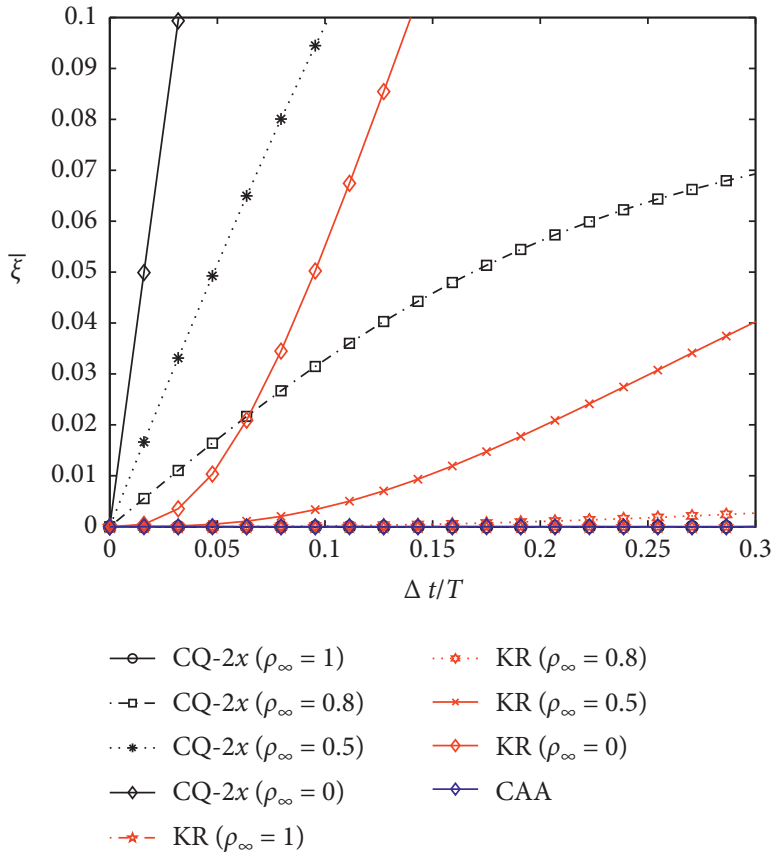

Figure 6: Numerical damping ratio of CQ- $2 x$ when $\xi=0$.

Substituting equation (56) into equation (57) yields a value for $u_{\Delta t}$ as follows:

$$
\begin{aligned}
\left(m+\frac{1}{2} c \Delta t+\frac{(x+1)^{2}}{4} k_{0} \Delta t^{2}\right) u_{\Delta t}= & \left(m+\frac{1}{2} c \Delta t+\frac{x^{2}+2 x-3}{4} k_{0} \Delta t^{2}\right) u_{0} \\
& +\left(m-\frac{1}{2} c \Delta t+\frac{(x-1)^{2}}{4} k_{0} \Delta t^{2}\right) \Delta t v_{0}+\frac{1}{2}\left(-m+\frac{1}{2} c \Delta t-\frac{(x-1)^{2}}{4} k_{0} \Delta t^{2}\right) \Delta t^{2} a_{0}+f_{0} \Delta t^{2}
\end{aligned}
$$

With known initial values of $u_{0}, v_{0}$, and $a_{0}$, the value of $u_{\Delta t}$ can be calculated using equation (58). Thus, a selfstarting displacement calculation procedure for CQ- $2 x$ can be obtained.

When the velocity is to be calculated, equation (59) can be used, with a second-order time accuracy of velocity:

$$
v_{\Delta t}=\frac{3 u_{\Delta t}-3 u_{0}}{\Delta t-2 v_{0}}-\frac{1}{2} a_{0} \Delta t .
$$

Equation (59) represents the self-starting velocity calculation in CQ- $2 x$.
When $\Omega_{0} \longrightarrow \infty$, equation (58) becomes

$$
\begin{aligned}
\lim _{\Omega_{0} \rightarrow \infty} u_{\Delta t}= & \frac{x^{2}+2 x-3}{(x+1)^{2}} u_{0}+\frac{(x-1)^{2}}{(x+1)^{2}} \Delta t v_{0} \\
& -\frac{1}{2} \frac{(x-1)^{2}}{(x+1)^{2}} \Delta t^{2} a_{0} .
\end{aligned}
$$

For CQ-2x, as expressed in equation (46), the recursive relationship among $u_{t+2 \Delta t}, u_{t+\Delta t}$, and $u_{t}$ can be expressed as follows: 


$$
\lim _{\Omega \longrightarrow \infty} u_{t+2 \Delta t}=\frac{2(x-1)}{1+x} u_{t+\Delta t}-\frac{(1-x)^{2}}{(1+x)^{2}} u_{t} .
$$

It can be seen from equations (60) and (61) that CQ-2x has zero-order displacement overshoot. Similarly, it is easy to find that CQ- $2 x$ has zero-order velocity overshoot by considering equations (21) and (59). Thus, CQ- $2 x$ can be classified as a family of noniterative algorithms of the form $U_{0} V_{0}\left(\rho_{\infty}, \rho_{\infty}\right)[20]$.

5.7. Calculation Procedure for MDOF Systems. When applied in MDOF systems, the forms of the aforementioned equations have some changes. When applied in MDOF systems, $m, c_{t+i \Delta t}$, and $k_{t+i \Delta t}$ appearing in a SDOF system should then be replaced by matrices $M, C_{t+i \Delta t}$, and $K_{t+i \Delta t} ; u_{t+i \Delta t}, v_{t+i \Delta t}$, $a_{t+i \Delta t}$, and $f_{t+i \Delta t}$ appearing in a SDOF system should be replaced by vectors $\left\{u_{t+i \Delta t}\right\},\left\{v_{t+i \Delta t}\right\},\left\{a_{t+i \Delta t}\right\}$, and $\left\{f_{t+i \Delta t}\right\}$. The calculation procedure for MDOF systems is provided as follows:

(1) Formulate the stiffness matrix $K_{0}$, mass matrix $M$, and damping matrix $C_{0}$ of the systems.

(2) Select appropriate coefficient $\rho_{\infty}$ and time increment $\Delta t$. Determine $x$ by solving equation (49d).

(3) Obtain initial values $\left\{u_{0}\right\}$ and $\left\{v_{0}\right\}$. Calculate $\left\{a_{0}\right\}$ by

$$
a_{0}=M^{-1}\left(\left\{f_{0}\right\}-C_{0}\left\{v_{0}\right\}-K_{0}\left\{u_{0}\right\}\right) .
$$

(4) Calculate $\left\{u_{\Delta t}\right\}$ and $\left\{v_{\Delta t}\right\}$ by equations (58) and (59), respectively. Then, calculate nonlinear stiffness and damping $K_{\Delta t}$ and $C_{\Delta t}$ by equation (4). Subsequently, calculate $\left\{a_{\Delta t}\right\}$ by the following equation:

$$
a_{t+\Delta t}=\frac{1}{m}\left(f_{t+\Delta t}-c_{t+\Delta t} v_{t+\Delta t}-k_{t+\Delta t} u_{t+\Delta t}\right) .
$$

(5) For each time step $(i=2,4,5, \ldots, n)$, calculate $\left\{u_{i \Delta t}\right\}$ and $\left\{v_{i \Delta t}\right\}$ by equations (49a) and (49b), respectively. Then, calculate nonlinear stiffness and damping $K_{i \Delta t}$ and $C_{i \Delta t}$ by equation (4). Subsequently, calculate $\left\{a_{i \Delta t}\right\}$ by equation (49c).

The main calculation efforts of CQ-2x are concentrated in Step 5 discussed above. The main calculation efforts in Step 5 are concentrated in equation (49a) because the coefficient matrix $\left(M+(1 / 2) C_{t+\Delta t} \Delta t+\left((x+1)^{2} / 4\right) K_{t+\Delta t} \Delta t^{2}\right)$ in equation (49a) is nondiagonal and solving the coupling linear equations needs $\vartheta\left(N^{3}\right)$ times of multiplication or division. The computational costs consumed by equation (49b) are $\vartheta(N)$, and those consumed by equations (4) and (3) are $\vartheta\left(N^{2}\right)$ since solving equation (4) only needs vector calculations and solving equation (3) only needs solving linear decoupling equations whose coefficient matrix is the diagonal matrix $M$. Thus, it is true that there is only one solver of equation (49a) within one time step for CQ- $2 x$. Furthermore, when no velocity and acceleration need to be calculated, the entire calculation of CQ- $2 x$ can be completed with only the involvement of displacement. In this case, the time consumption of CQ-2x will become less, and the storage requirements of CQ- $2 x$ will become considerably lower than other unconditionally stable noniterative algorithms.

\section{Numerical Examples}

To evaluate the overall behavior and confirm the convergence rate, controllable numerical dissipation, unconditional stability in case of stiffness hardening, and computational efficiency of the derived algorithms, four examples are given. The CAA method, as an example of an implicit algorithm, and the KR- $\alpha$ method, as an example of a family of unconditionally stable structure-dependent noniterative algorithms, are considered for comparison.

6.1. Example 1. The purpose of this example is to validate the convergence rate of the CQ-2x method. Consider a singleDOF linear resonance problem with the initial conditions $u(0)=v(0)=1$ :

$$
a(t)+\omega^{2} u(t)=\sin (\omega t)
$$

The exact solutions [27] to this problem are

$$
\begin{aligned}
& u(t)=\frac{2 \omega+1}{2 \omega^{2}} \sin (\omega t)+\frac{2 \omega-t}{2 \omega} \cos (\omega t), \\
& v(t)=\frac{t-2 \omega}{2} \sin (\omega t)+\cos (\omega t), \\
& a(t)=\frac{1-2 \omega}{2} \sin (\omega t)+\omega \frac{t-2 \omega}{2} \cos (\omega t) .
\end{aligned}
$$

The circular self-frequency $\omega$ was assigned a value of $2 \pi$. Thus, the period is $T=1 \mathrm{~s}$. The integration interval was chosen to be $T_{\text {Fin }}=10 T=10 \mathrm{~s}$. In the calculations, the step size $\Delta t$ was varied from $10^{-1} \mathrm{~s}$ to $10^{-4} \mathrm{~s}$. The plots in Figures 7-12 show the curves of the maximum relative absolute errors and average relative absolute errors in displacement, velocity, and acceleration for the duration of the simulation. These errors were computed as functions of $\Delta t$ (on a logarithmic scale) using the CQ- $2 x$, CAA, and KR- $\alpha$ methods. When $\rho_{\infty} \neq 1$, the CQ- $2 x$ method shows first-order rates of convergence in displacement, velocity, and acceleration, identical to the KR- $\alpha$ method. When $\rho_{\infty}=1, \mathrm{CQ}-2 x$ shows second-order rates of convergence in displacement, velocity, and acceleration, identical to the CAA method.

6.2. Example 2. The purpose of this example is to validate the controllable numerical dissipation and unconditional stability in case of stiffness hardening of the CQ- $2 x$ method. In this example, the well-known unforced and undamped Duffing oscillator [28] is considered. The nonlinear dynamics equation is

$$
a+S_{1} u\left(1+S_{2} u^{2}\right)=0
$$

where $S_{1}=100$ and $S_{2}-10$. The initial conditions are $u_{0}=$ 1.5 and $v_{0}=0$. 


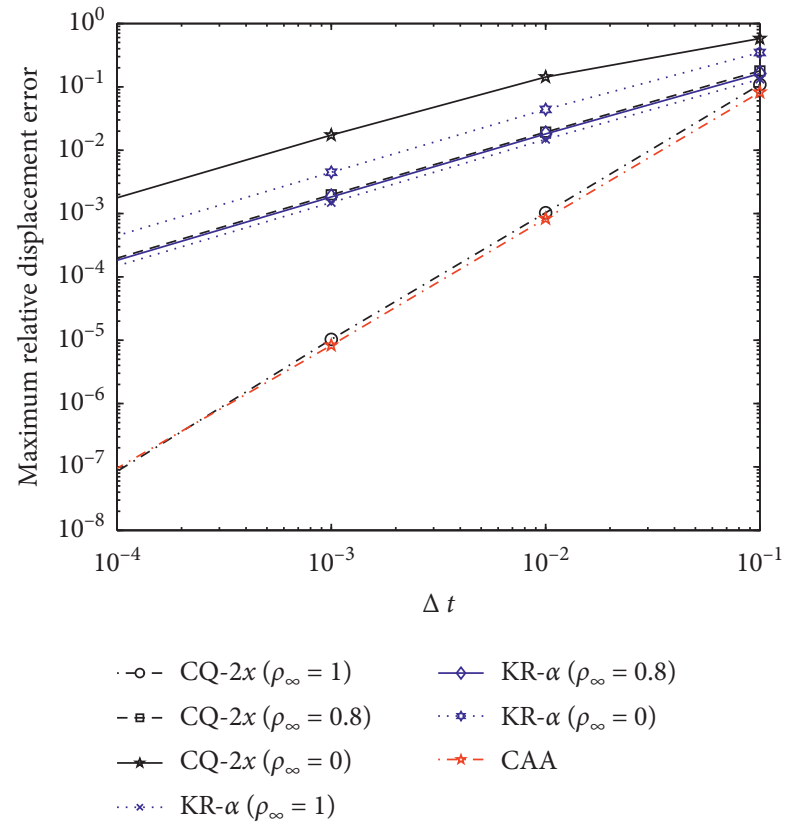

FIgURE 7: Convergence rate of CQ-2x assessed in terms of the maximum relative displacement error.

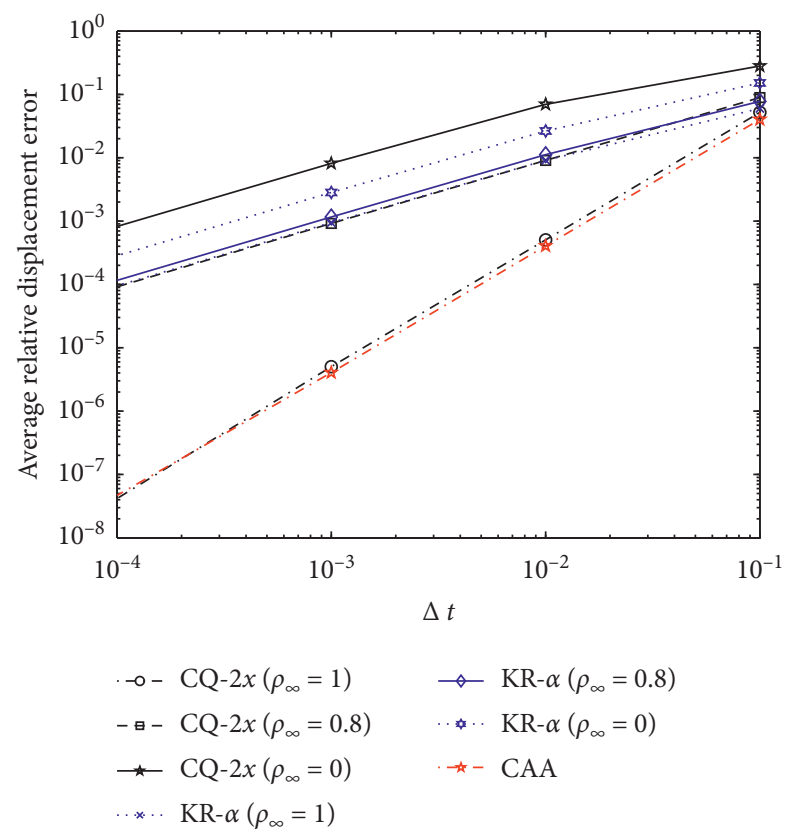

FIGURE 8: Convergence rate of CQ- $2 x$ assessed in terms of the average relative displacement error.

Equation (66) represents a hardening elastic spring. This is an example of a conservative system that maintains a constant total energy $E$ in its exact solutions. For equation (66),

$$
E=\frac{1}{2} v^{2}+\frac{1}{2} S_{1} u^{2}+\frac{1}{4} S_{1} S_{2} u^{4}
$$

To assess the time integration scheme, the percentage error in terms of the energy is introduced [29]:

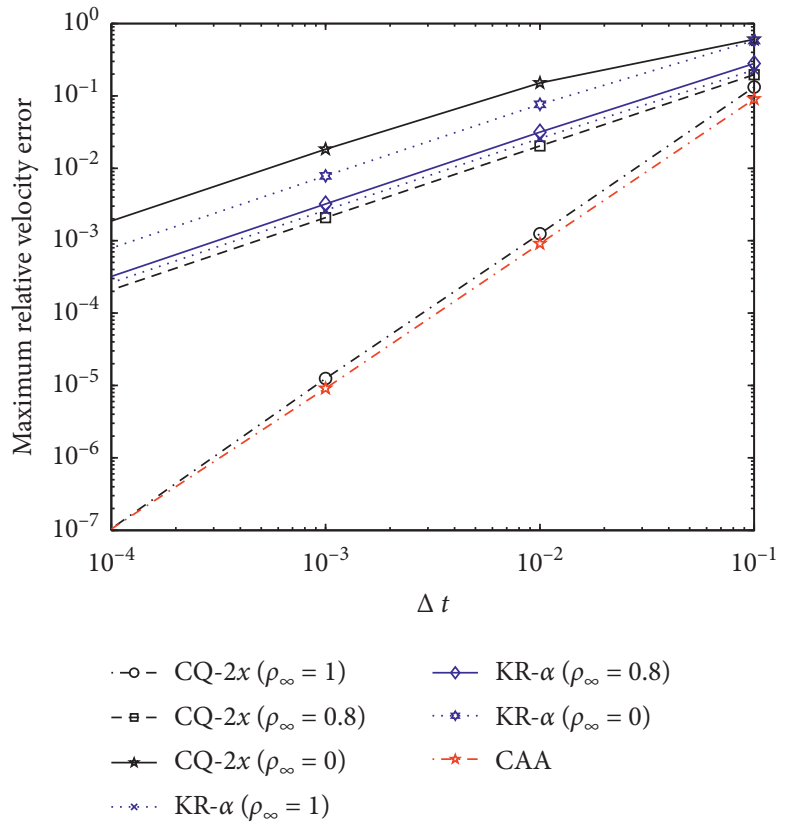

FIgURE 9: Convergence rate of CQ- $2 x$ assessed in terms of the maximum relative velocity error.

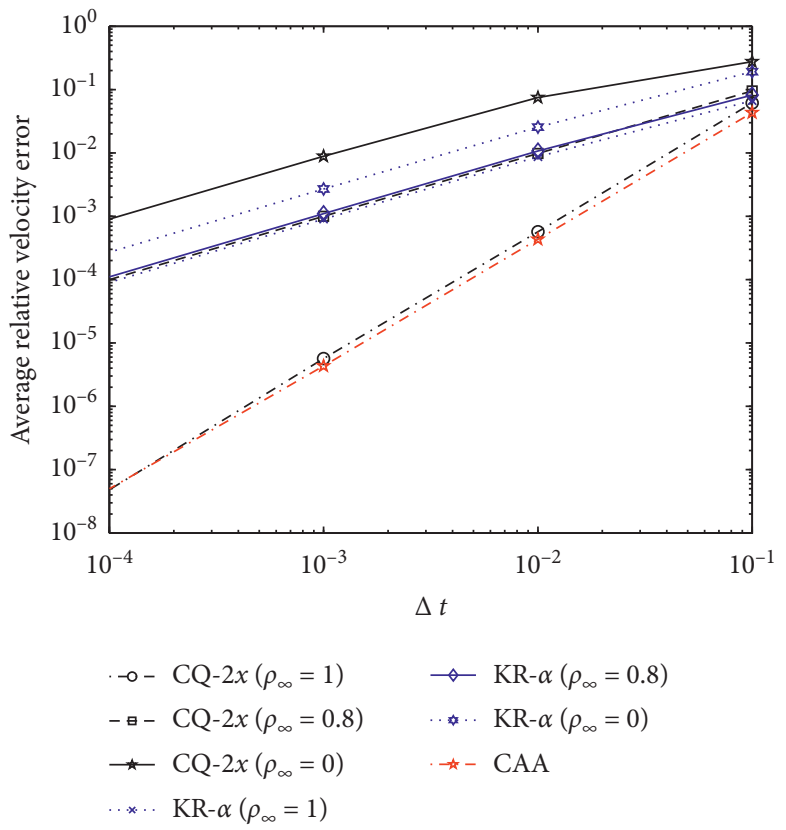

Figure 10: Convergence rate of CQ-2x assessed in terms of the average relative velocity error.

$$
E_{r}=\left|\frac{E-E_{0}}{E_{0}}\right| \times 100 \%
$$

where $E_{0}$ is the total energy at $t=0$.

Equation (66) has an exact solution expressed in terms of Jacobian elliptic functions [30]:

$$
u(t)=u_{0} c n(-\widehat{w} t, k),
$$




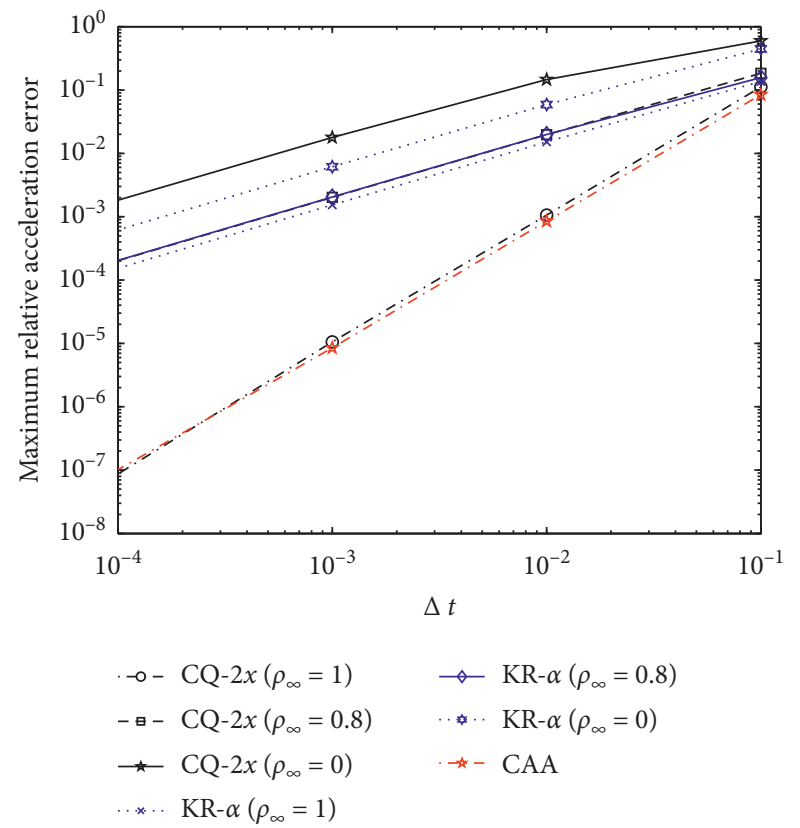

FIgURE 11: Convergence rate of CQ-2x assessed in terms of the maximum relative acceleration error.

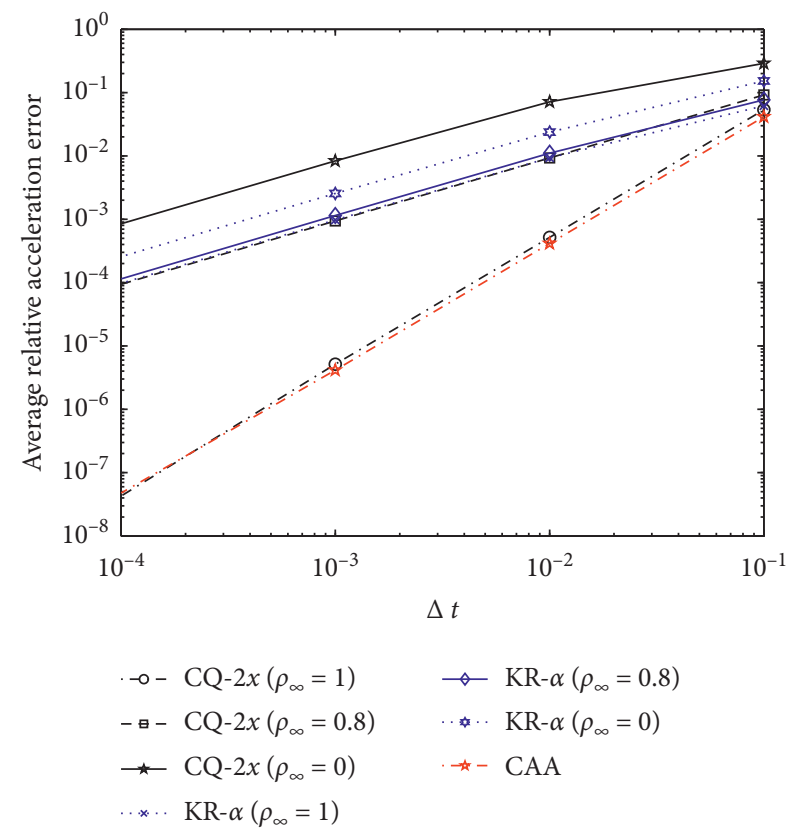

FIgURE 12: Convergence rate of CQ-2x assessed in terms of the average relative acceleration error.

where

$$
\begin{aligned}
& \widehat{\omega}^{2}=S_{1}\left(1+S_{2} u_{0}^{2}\right), \\
& k^{2}=\frac{S_{2} u_{0}^{2}}{2\left(1+S_{2} u_{0}^{2}\right)} .
\end{aligned}
$$

The period $T$ of this oscillator is equal to 0.15 . The exact phase portrait is given in Figure 13. Also, shown in
Figures 14-22 are the phase portraits of numerical results obtained using different integration schemes with the same time step size of $\Delta t=T / 20$ over a time duration of $100 \mathrm{~T}$. The maximum values of the percentage errors of the nine considered algorithms over this time duration of $100 \mathrm{~T}$ are summarized in Table 1 for various time step sizes. When $\Delta t=(1 / 25) T$, CQ-2x $\left(\rho_{\infty}=1\right)$ has an error of $6.06 \%$, whereas the KR- $\alpha$ method has an error of at least $99.63 \%$. It can be seen from Figures 14-17 that $\rho_{\infty}$ is smaller, the numerical damping is bigger, and the undamped Duffing oscillator decays faster. To validate the unconditional stability of CQ- $2 x$ in the stiffness hardening case, a time step size of $\Delta t=(1 / 2) T$ over a time duration of $100 \mathrm{~T}$ is considered. Figures 23-31 show the phase portraits of the numerical results obtained using the nine integration schemes with the same time step size of $\Delta t=(1 / 2) T$ over a time duration of $100 \mathrm{~T}$. It is meaningless to study an algorithm's accuracy when $\Delta t=(1 / 2) T$, but it is useful to explore its stability. Figures $23-26$ show that even when $\Delta t=(1 / 2) T$, CQ-2x $\left(\rho_{\infty}=1,0.8,0.5,0\right)$ is still stable. By contrast, the KR$\alpha$ method is robustly stable only when $\rho_{\infty}=0$, as seen in Figure 30; when $\rho_{\infty}=1,0.8$, and 0.5 , the KR- $\alpha$ method becomes evidently unstable, as seen in Figures 27-29. e CAA method also exhibits some instability, as seen in Figure 31 (for the CAA method, the iteration termination criterion is that the absolute value of the error between the displacements in the previous and current iterations is less than $10^{-6}$ ).

The time consumptions of the different algorithms with different time steps over the time duration of $100 \mathrm{~T}$ are listed in Table 2. For the same time step size, the noniterative algorithms (CQ- $2 x$ and KR- $\alpha$ ) are more efficient than the implicit algorithm (CAA). In addition, the CQ- $2 x$ method is more efficient than the KR- $\alpha$ method for the same time step size. For example, when $\Delta t=(1 / 25) T$, the time consumption of CQ- $2 x$ is at most 0.0015 seconds, whereas the KR- $\alpha$ method requires at least 0.0048 seconds.

6.3. Example 3. The purpose of this example is to validate the efficiency of the CQ- $2 x$ method compared with the implicit method and $\mathrm{KR}-\alpha$ method. In this example, the forced vibration response of a $n$-DOF $(n=200,500,1000,2000$, and 4000) spring-mass system was considered, as shown in Figure 32. The structural properties of this system are assumed to be $m_{i}=100 \mathrm{~kg}$ and $k_{i}=10^{7}\left[1-\left(u_{i}-u_{i-1}\right)^{2}\right] \mathrm{N} / \mathrm{m}$, in which $i=1,2, \ldots, n$. This system is subjected to a ground acceleration of $10 \sin (\pi t)$. The highest natural frequency is $632.4 \mathrm{rad} / \mathrm{s}$ [31]. According to the highest natural frequency, a time step duration of $\Delta t=0.001 \mathrm{~s}$ is obtained from Newmark's average acceleration method and is selected to be the exact solution. The responses of displacement and velocity versus time of the 200th DOF when $N=200$ are plotted in Figures 33 and 34 . The analysis is accomplished by considering the time step duration equal to $0.02 \mathrm{~s}$. The maximum displacement and velocity error ratios of the 200th DOF when $n=200$ are listed in Table 3. It can be seen that the accuracy of the CQ- $2 x$ method is sufficient. The maximum error ratio here is defined by the function of max 


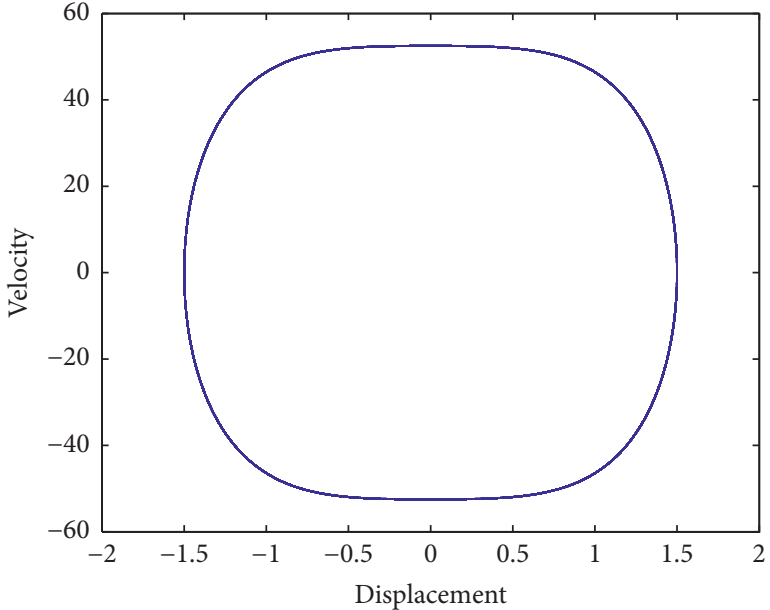

- Exact

FIGURE 13: Exact solution for the phase portrait of the hardening spring.

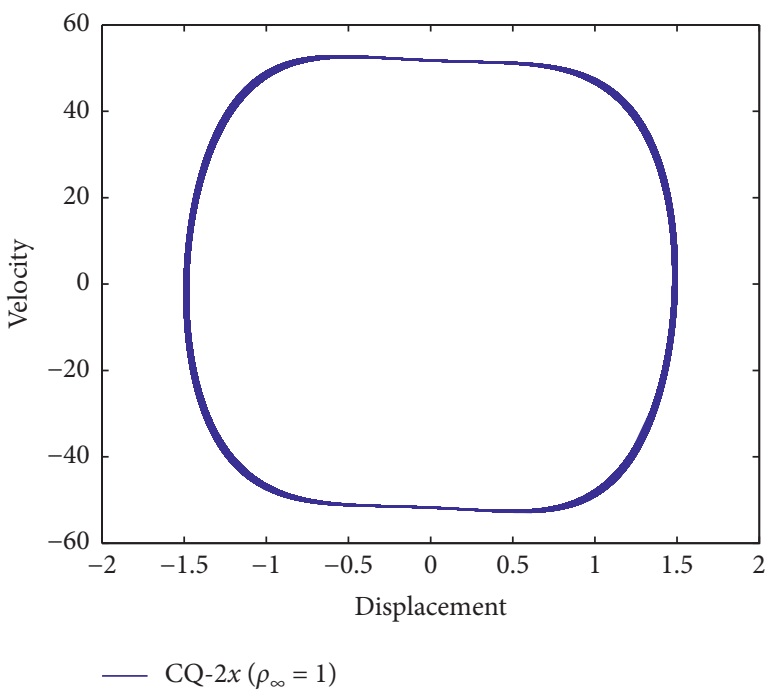

FIGURE 14: Phase portrait of the hardening spring calculated using CQ-2x $\left(\rho_{\infty}=1\right)$ when $\Delta t=T / 20$.

(abs(calculated value-exact value $)) / \max (\operatorname{abs}($ exact value $))$, where $\max =$ maximum and $\mathrm{abs}=$ absolute. Importantly, without the need for iteration calculations, CQ- $2 x$ algorithms have approximately less than half the computational time consumption compared to that consumed by the implicit algorithm CAA. With solving the inverse matrix only once for each time step, CQ- $2 x$ algorithms are more efficient than the noniterative $\mathrm{KR}-\alpha$ method in which solving the inverse matrix twice for each time step is needed, as listed in Table 4.

6.4. Example 4. The purpose of this example is to validate the efficiency of the CQ- $2 x$ method in case of Vehicle-Bridge Coupled Vibration where the contact nonlinearity exists. In

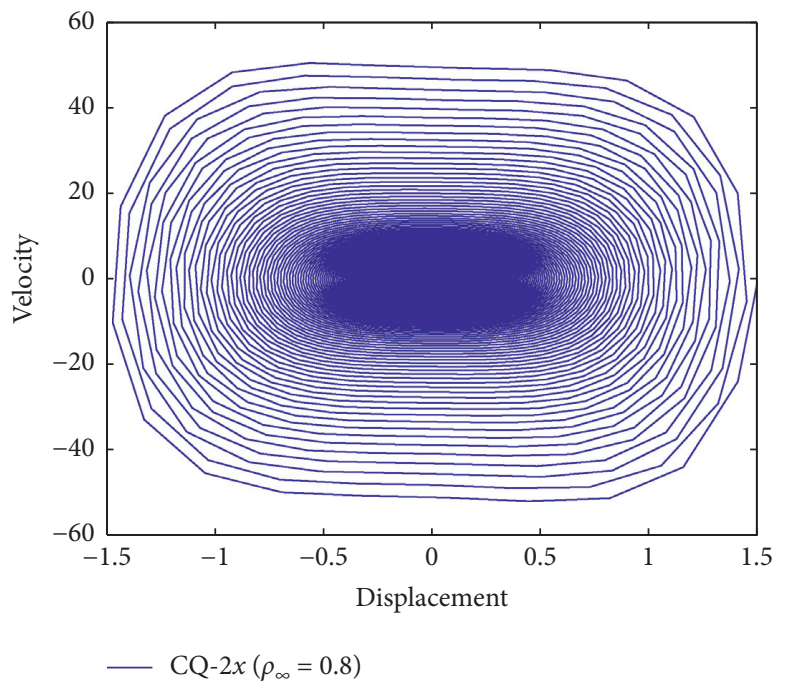

FIGURE 15: Phase portrait of the hardening spring calculated using CQ- $2 x\left(\rho_{\infty}=0.8\right)$ when $\Delta t=T / 20$.

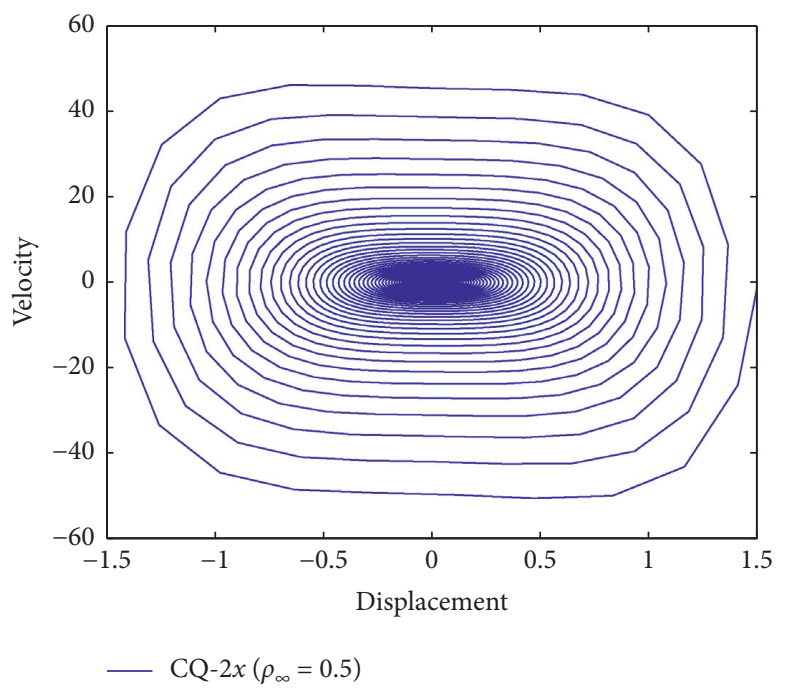

FIgURE 16: Phase portrait of the hardening spring calculated using CQ- $2 x\left(\rho_{\infty}=0.5\right)$ when $\Delta t=T / 20$.

this example, a simple beam of span length $=25 \mathrm{~m}$ is subjected to a moving spring mass [32] (as shown in Figure 35). The following data are adopted: Young's modulus $E=2.87 \mathrm{Gpa}, \rho=0.2$, moment of inertia $I=2.90 \mathrm{~m}^{4}$, mass per unit length $m=2303 \mathrm{~kg} / \mathrm{m}$, suspended mass $M_{v}=5750 \mathrm{~kg}$, suspension stiffness $k_{v}=1595 \mathrm{kN} / \mathrm{m}$, and speed $v=90 \mathrm{~km} / \mathrm{h}$. The frequency of vibration computed of the spring mass is $\omega_{v}=16.66 \mathrm{rad} / \mathrm{s}$. The frequencies of vibration computed of the bridge are $\omega_{1}=30.02 \mathrm{rad}$ $/ \mathrm{s}, \omega_{2}=120.08 \mathrm{rad} / \mathrm{s}, \omega_{3}=270.19 \mathrm{rad} / \mathrm{s}, \omega_{4}=480.34 \mathrm{rad} / \mathrm{s}$, $\omega_{5}=750.58 \mathrm{rad} / \mathrm{s}$, and $\omega_{6}=1081.0 \mathrm{rad} / \mathrm{s}$. The maximum frequency of vibration computed of the bridge is $\omega_{\max }=95432 \mathrm{rad} / \mathrm{s}$. According to stability requirement of the CDM, the widest time step satisfying CDM's stability is $2 / \omega_{\max }=2.0957 \times 10^{-5} \mathrm{~s}$. A time step duration of $\Delta t=2 \times$ 


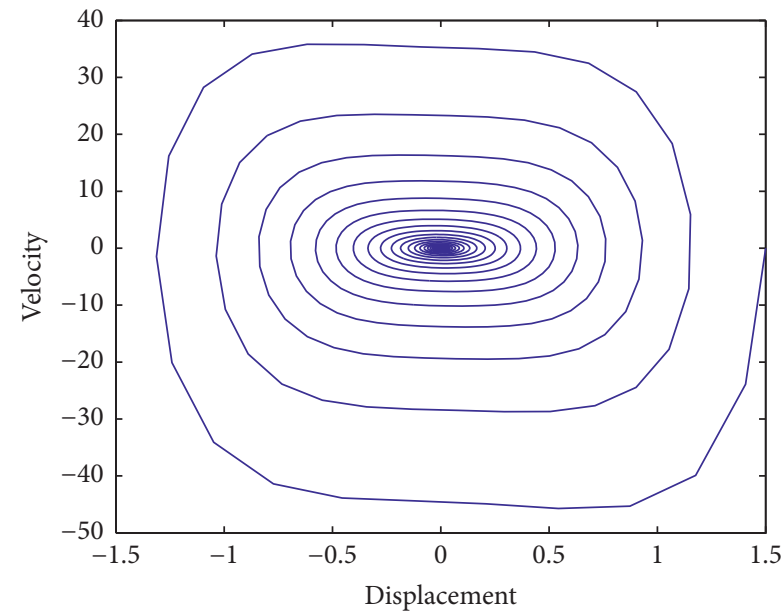

CQ- $2 x\left(\rho_{\infty}=0\right)$

FIGURE 17: Phase portrait of the hardening spring calculated using CQ-2x $\left(\rho_{\infty}=0\right)$ when $\Delta t=T / 20$.

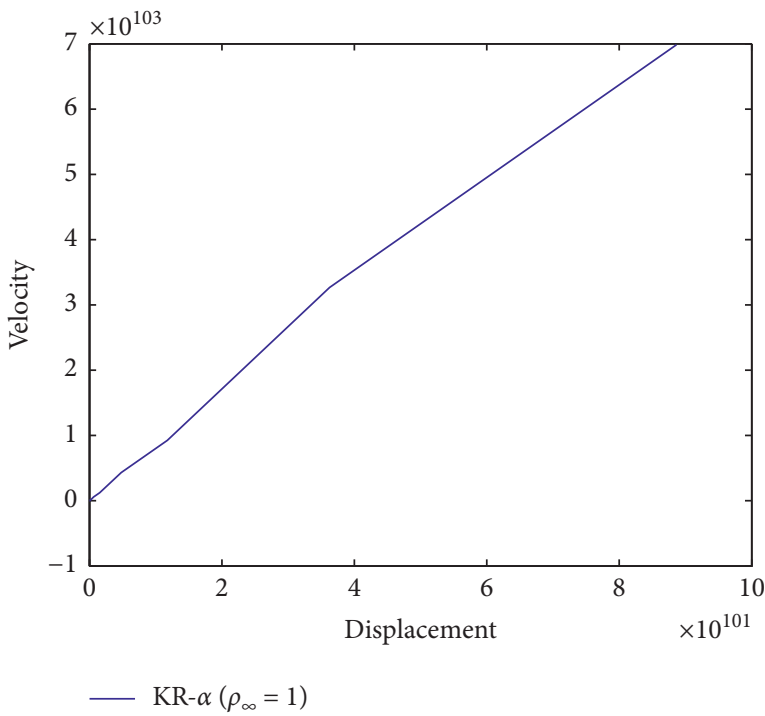

FIGURE 18: Phase portrait of the hardening spring calculated using the KR- $\alpha$ method $\left(\rho_{\infty}=1\right)$ when $\Delta t=T / 20$.

$10^{-5} \mathrm{~s}$ is obtained for the CDM, and the calculation result is selected to be the exact solution, while with unconditional stability, the time step duration of CQ2x $\left(\rho_{\infty}=1\right)$, KR$\alpha\left(\rho_{\infty}=1\right)$, and CAA is only determined by the requirements of the calculation accuracy; here, $\Delta t=0.001 \mathrm{~s}$. The midpoint vertical deflection of the beam and deflection of the spring mass versus time are plotted in Figures 36 and 37. The maximum displacement error ratios (defined as that in Example 3) of the midpoint vertical deflection of the beam and the deflection of the spring mass are listed in Table 5. It can be seen that the accuracy of the CQ- $2 x$ method is sufficient. The efficiency of the CDM $(\Delta t=0.00002 \mathrm{~s})$,

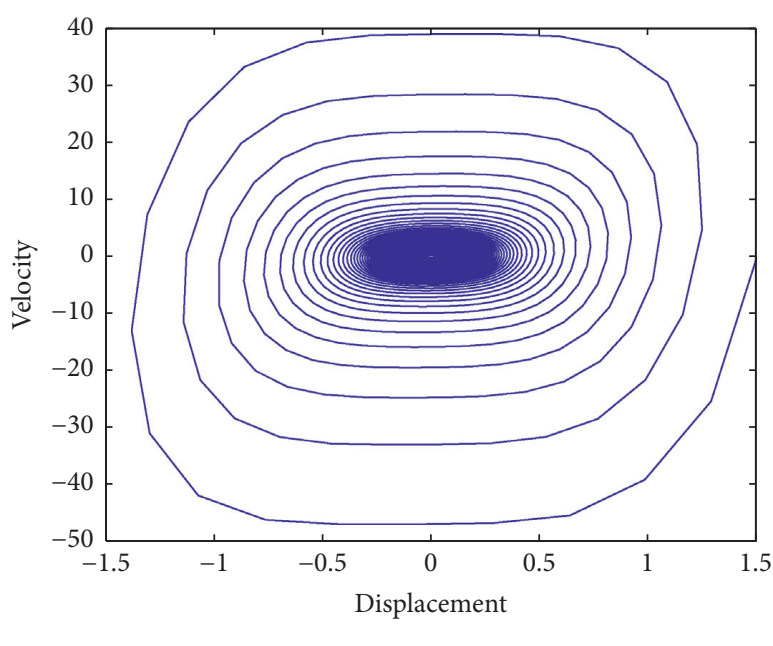

KR- $\alpha\left(\rho_{\infty}=0.8\right)$

FIGURE 19: Phase portrait of the hardening spring calculated using the KR- $\alpha$ method $\left(\rho_{\infty}=0.8\right)$ when $\Delta t=T / 20$.

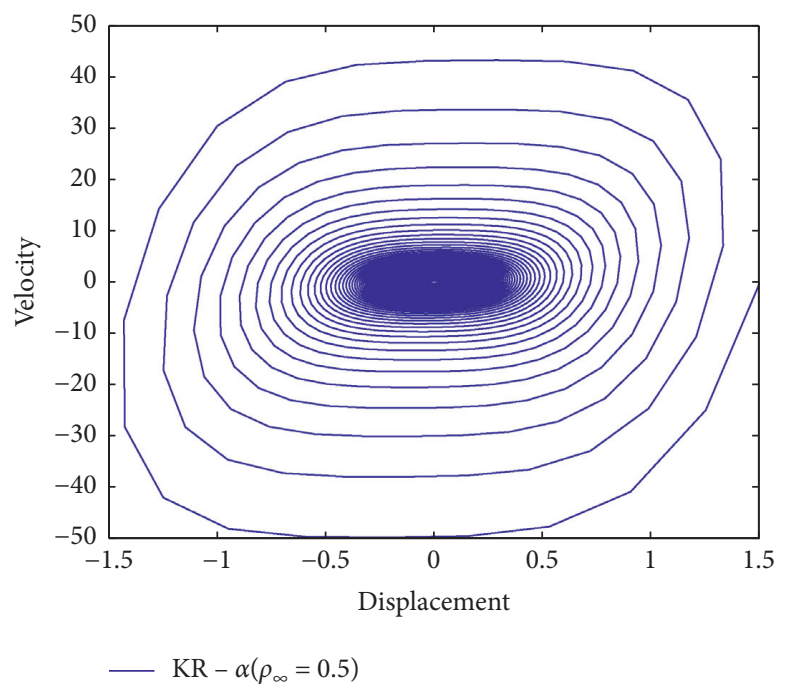

FIgURE 20: Phase portrait of the hardening spring calculated using the KR- $\alpha$ method $\left(\rho_{\infty}=0.5\right)$ when $\Delta t=T / 20$.

$\operatorname{CQ} 2 x\left(\rho_{\infty}=1\right), \operatorname{KR}-\alpha\left(\rho_{\infty}=1\right)$, and CAA $(\Delta t=0.001 \mathrm{~s})$ is compared. The average time consumption of the four algorithms in which every algorithm had been performed five times is listed in Table 6. In this example, limited by its conditional stability, the CDM has to be performed with very small $\Delta t=0.00002 \mathrm{~s}$, which leads to its efficiency much lower than the other three algorithms. Without the need for iteration calculations, CQ- $2 x$ and KR- $\alpha$ algorithms show higher efficiency than that of the implicit algorithm CAA although the maximum number of iteration in each time step of CAA is only 3 . The CQ- $2 x$ algorithm are more efficient than the noniterative $\mathrm{KR}-\alpha$ algorithm since the computation amount in each time step of CQ-2x is much less than that of KR- $\alpha$. 


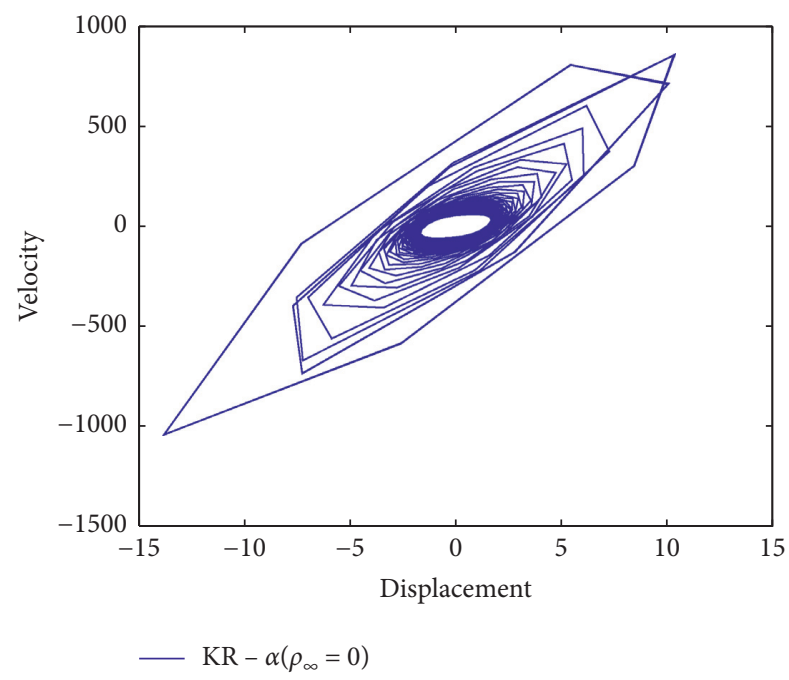

FIGURE 21: Phase portrait of the hardening spring calculated using the KR- $\alpha$ method $\left(\rho_{\infty}=0\right)$ when $\Delta t=T / 20$.

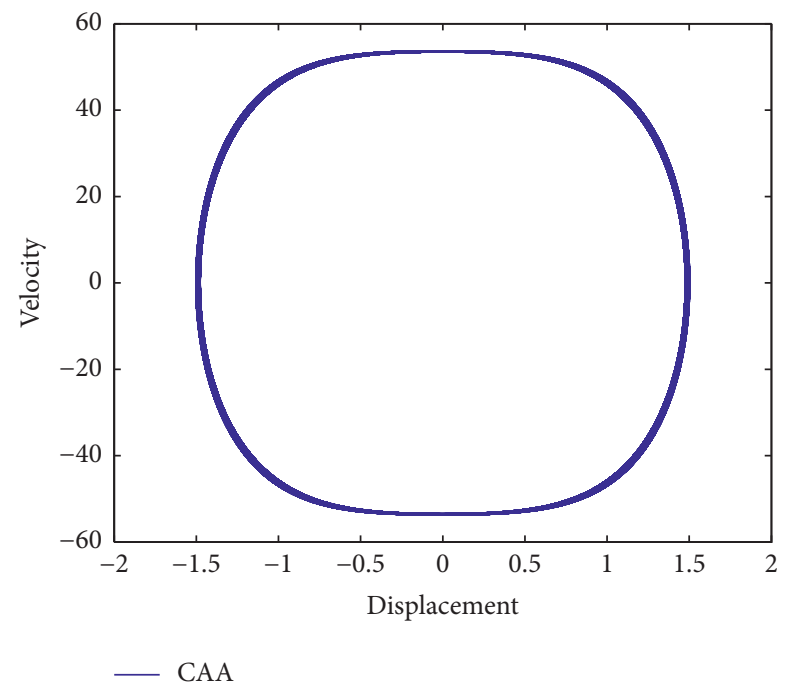

FIGURE 22: Phase portrait of the hardening spring calculated using the CAA method when $\Delta t=T / 20$.

TABLE 1: Maximum percentage errors of the total energy over a time duration of $100 T$ for the hardening spring.

\begin{tabular}{|c|c|c|c|c|c|c|c|c|c|}
\hline \multirow[b]{2}{*}{$\Delta t$} & \multicolumn{4}{|c|}{ CQ- $2 x$} & \multicolumn{4}{|c|}{$\mathrm{KR}-\alpha$} & \multirow{2}{*}{ CAA } \\
\hline & $\rho_{\infty}=1$ & $\rho_{\infty}=0.8$ & $\rho_{\infty}=0.5$ & $\rho_{\infty}=0$ & $\rho_{\infty}=1$ & $\rho_{\infty}=0.8$ & $\rho_{\infty}=0.5$ & $\rho_{\infty}=0$ & \\
\hline$T / 1000$ & 0.00 & 19.88 & 45.54 & 77.22 & 71.63 & 68.55 & 60.60 & 1.31 & 0.00 \\
\hline$T / 200$ & 0.09 & 60.80 & 87.93 & 98.24 & 96.00 & 95.22 & 92.97 & 6.83 & 0.04 \\
\hline$T / 100$ & 0.36 & 79.76 & 96.07 & 99.68 & 99.32 & 98.25 & 97.36 & 15.89 & 0.17 \\
\hline$T / 50$ & 1.47 & 92.13 & 99.08 & 99.97 & 158.39 & 99.35 & 99.04 & 37.14 & 0.66 \\
\hline$T / 25$ & 6.06 & 97.73 & 99.87 & 100.00 & 105670.02 & 99.74 & 99.63 & 400.59 & 2.62 \\
\hline$T / 20$ & 9.51 & 98.60 & 99.94 & 100.00 & $\infty$ & 99.81 & 99.73 & 700089.87 & 4.07 \\
\hline$T / 15$ & 16.77 & 99.28 & 99.98 & 100.00 & $\infty$ & 99.87 & 99.81 & 219047.46 & 7.13 \\
\hline$T / 2$ & Finite & Finite & Finite & Finite & $\infty$ & $\infty$ & $\infty$ & finite & $\infty$ \\
\hline
\end{tabular}




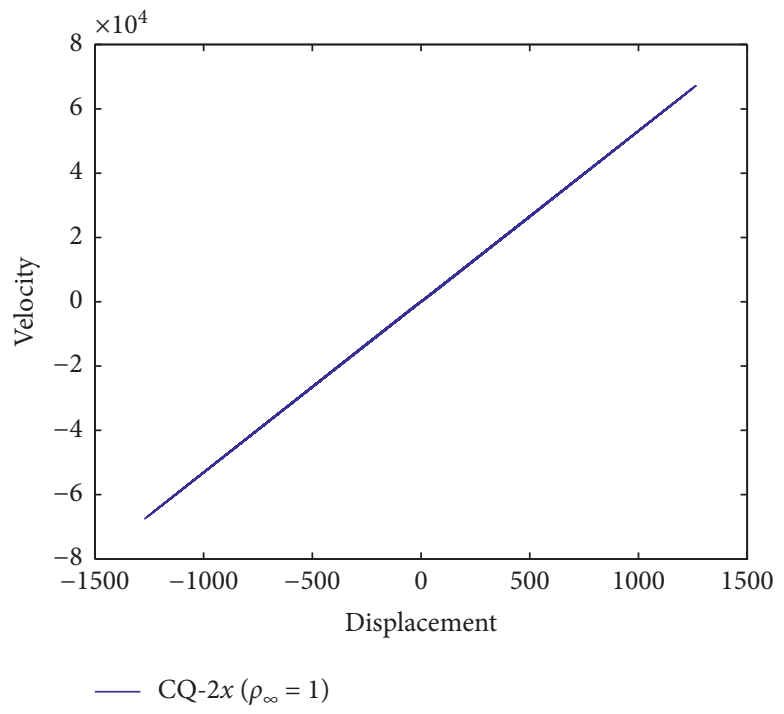

FIGURE 23: Phase portrait of the hardening spring calculated using CQ-2x $\left(\rho_{\infty}=1\right)$ when $\Delta t=(1 / 2) T$.

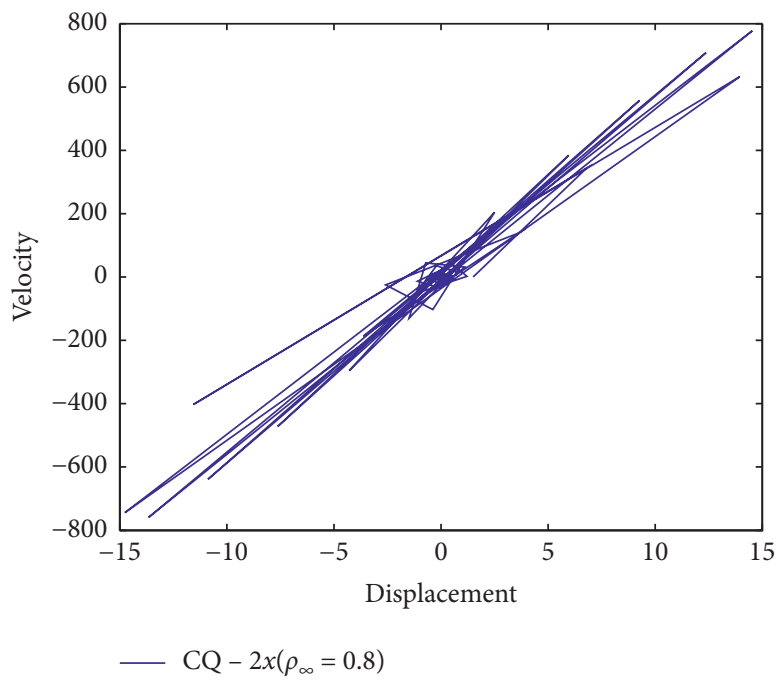

FIGURE 24: Phase portrait of the hardening spring calculated using CQ-2x $\left(\rho_{\infty}=0.8\right)$ when $\Delta t=(1 / 2) T$.

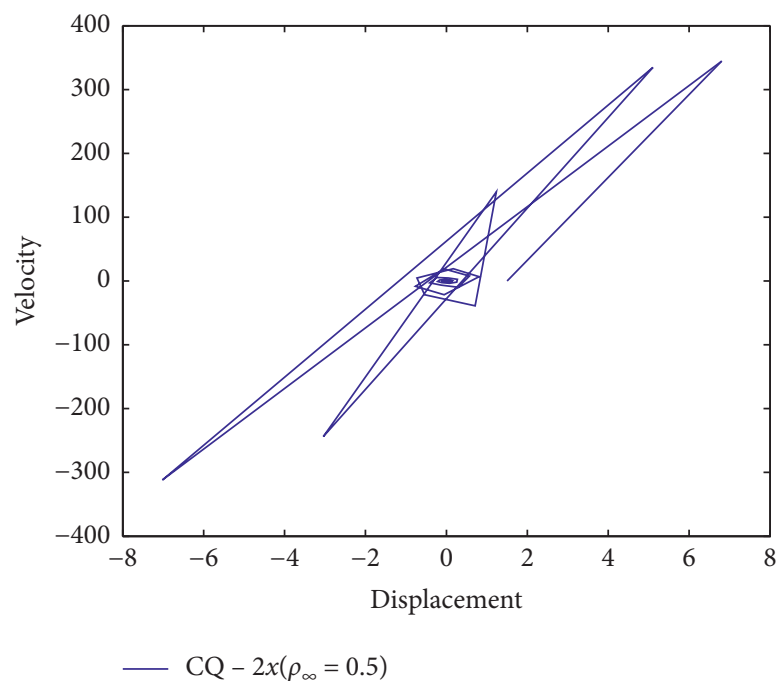

FIGURE 25: Phase portrait of the hardening spring calculated using CQ-2x $\left(\rho_{\infty}=0.5\right)$ when $\Delta t=(1 / 2) T$. 


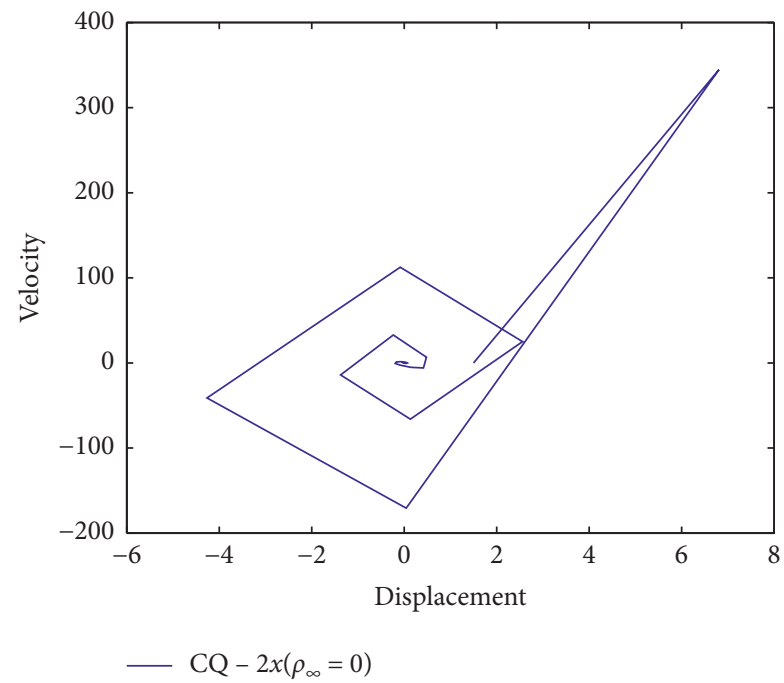

FIgURE 26: Phase portrait of the hardening spring calculated using CQ-2x $\left(\rho_{\infty}=0\right)$ when $\Delta t=(1 / 2) T$.

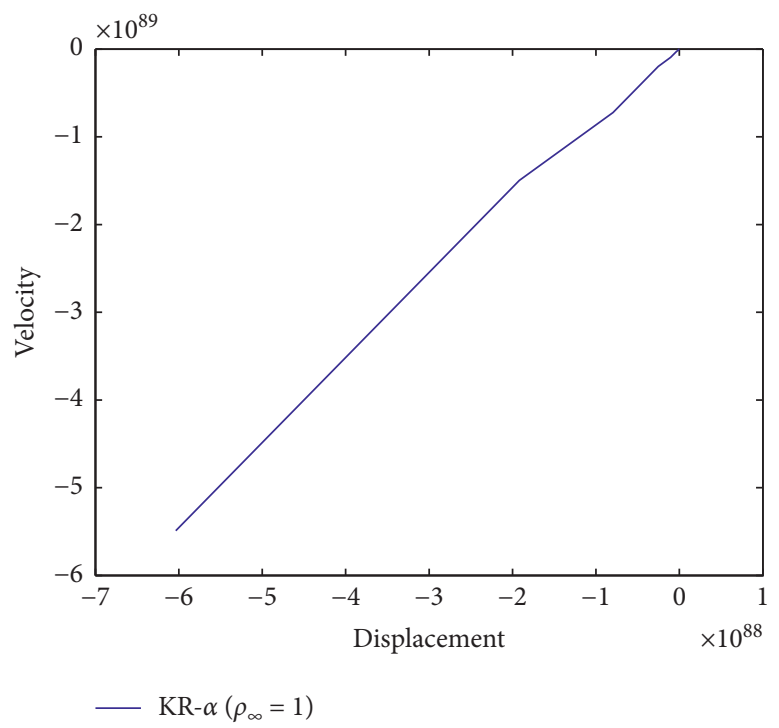

FIGURE 27: Phase portrait of the hardening spring calculated using the KR- $\alpha$ method $\left(\rho_{\infty}=1\right)$ when $\Delta t=(1 / 2) T$.

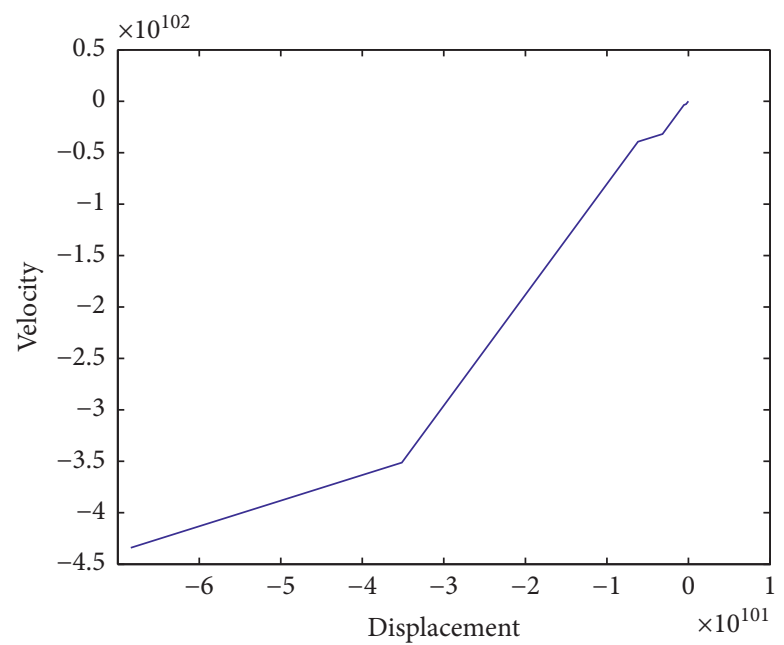

$-\operatorname{KR}-\alpha\left(\rho_{\infty}=0.8\right)$

FIgURE 28: Phase portrait of the hardening spring calculated using the KR-method $\left(\rho_{\infty}=0.8\right)$ when $\Delta t=(1 / 2) T$. 


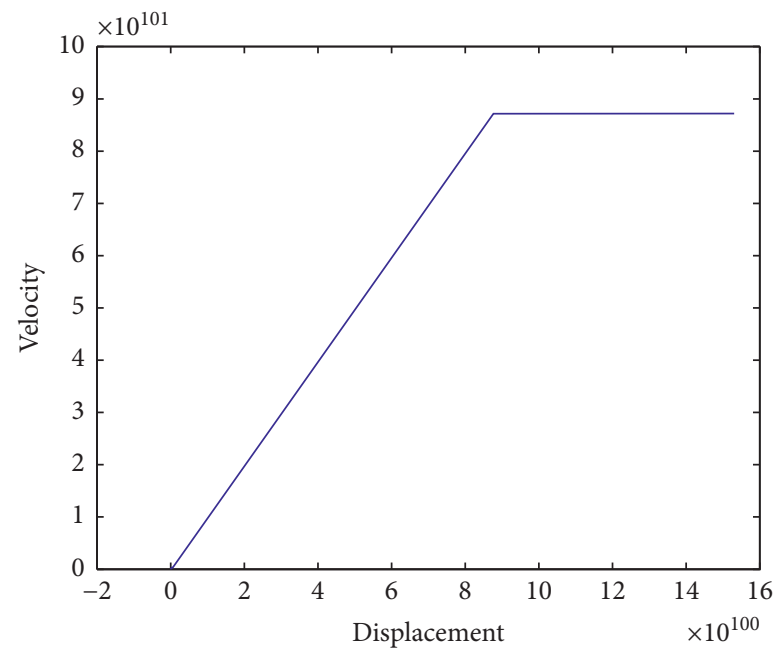

$-\operatorname{KR}-\alpha\left(\rho_{\infty}=0.5\right)$

FIGURE 29: Phase portrait of the hardening spring calculated using the KR- $\alpha$ method $\left(\rho_{\infty}=0.5\right)$ when $\Delta t=(1 / 2) T$.

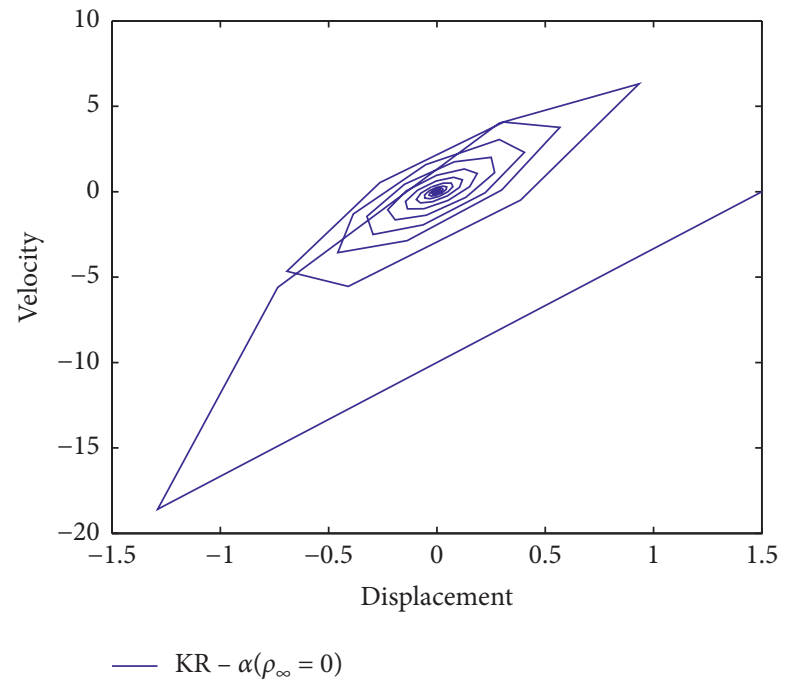

FIGURE 30: Phase portrait of the hardening spring calculated using the KR- $\alpha$ method $\left(\rho_{\infty}=0\right)$ when $\Delta t=(1 / 2) T$.

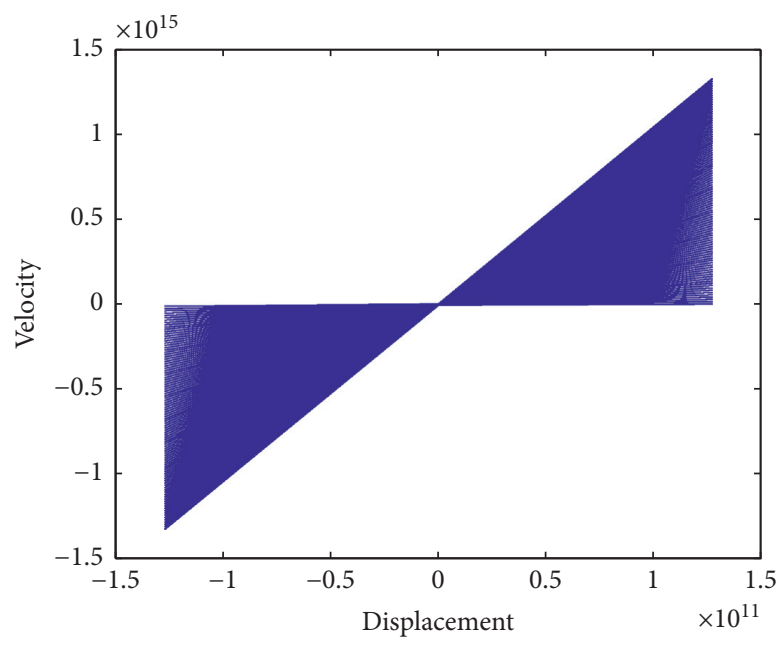

- CAA

FIgURE 31: Phase portrait of the hardening spring calculated using the CAA method when $\Delta t=(1 / 2) T$. 
TABLE 2: Time consumptions of the different algorithms for solving the Duffing vibration problem in Example 2 over a time duration of 100 $T$ (unit: second).

\begin{tabular}{|c|c|c|c|c|c|c|c|c|c|}
\hline \multirow[b]{2}{*}{$\Delta t$} & \multicolumn{4}{|c|}{ CQ- $2 x$} & \multicolumn{4}{|c|}{$\mathrm{KR}-\alpha$} & \multirow{2}{*}{ CAA } \\
\hline & $\rho_{\infty}=1$ & $\rho_{\infty}=0.8$ & $\rho_{\infty}=0.5$ & $\rho_{\infty}=0$ & $\rho_{\infty}=1$ & $\rho_{\infty}=0.8$ & $\rho_{\infty}=0.5$ & $\rho_{\infty}=0$ & \\
\hline$T / 1000$ & 0.0590 & 0.0090 & 0.0180 & 0.0174 & 0.3305 & 0.1871 & 0.1936 & 0.1947 & 1.1631 \\
\hline$T / 200$ & 0.0115 & 0.0018 & 0.0037 & 0.0036 & 0.0686 & 0.0405 & 0.0474 & 0.0431 & 0.2700 \\
\hline$T / 100$ & 0.0060 & 0.0009 & 0.0020 & 0.0019 & 0.0331 & 0.0199 & 0.0198 & 0.0241 & 0.1386 \\
\hline$T / 50$ & 0.0032 & 0.0004 & 0.0009 & 0.0009 & 0.0166 & 0.0095 & 0.0104 & 0.0125 & 0.0835 \\
\hline$T / 25$ & 0.0015 & 0.0002 & 0.0005 & 0.0005 & 0.0079 & 0.0048 & 0.0048 & 0.0080 & 0.0511 \\
\hline$T / 20$ & 0.0015 & 0.0002 & 0.0004 & 0.0004 & 0.0066 & 0.0040 & 0.0039 & 0.0068 & 0.0426 \\
\hline$T / 15$ & 0.0009 & 0.0001 & 0.0003 & 0.0003 & 0.0058 & 0.0027 & 0.0029 & 0.0051 & 0.0386 \\
\hline
\end{tabular}

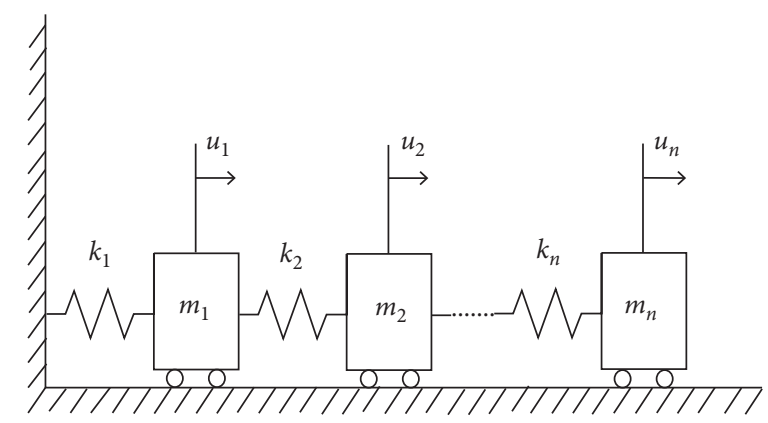

FIgURE 32: Schematic plot of the $n$-DOF spring-mass system.

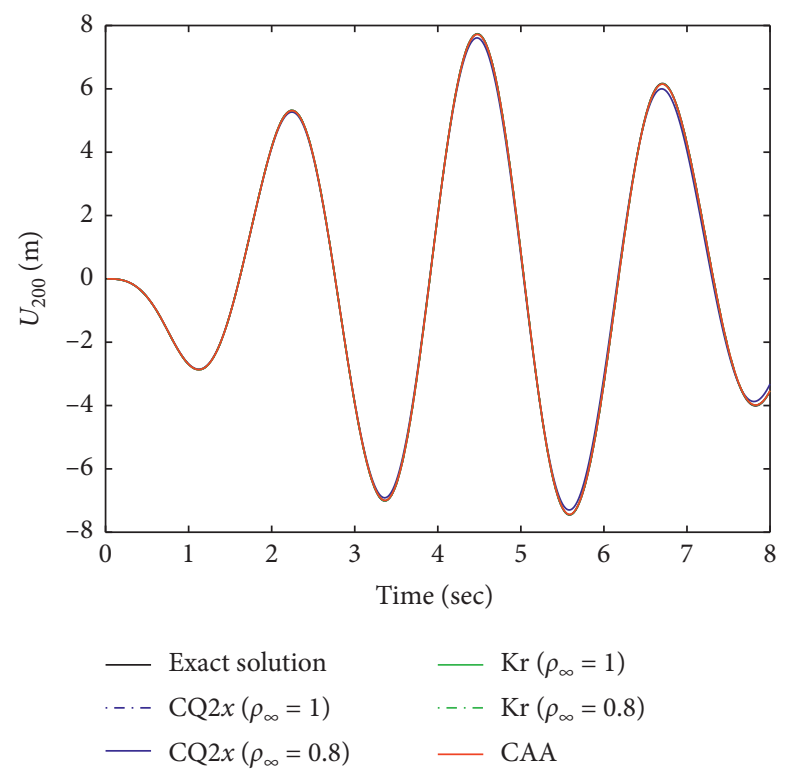

FIgURE 33: Displacement responses of the 200th DOF when $n=200$. 


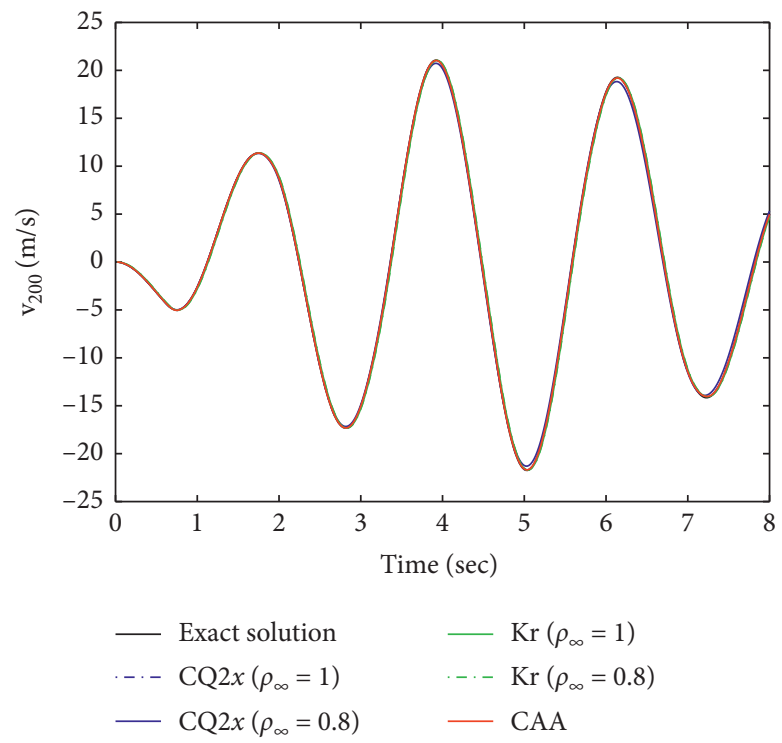

Figure 34: Velocity responses of the 200th DOF when $n=200$.

TABLE 3: Maximum displacement and velocity responses' errors of the 200th DOF when $n=200$.

\begin{tabular}{lccccc}
\hline & CAA & \multicolumn{2}{c}{ CQ-2x } & \multicolumn{2}{c}{ KR- $\alpha$} \\
& & $\rho_{\infty}=1$ & $\rho_{\infty}=0.8$ & $\rho_{\infty}=1$ & 0.00283 \\
\hline Displacement & 0.00331 & 0.00284 & 0.03117 & 0.00289 \\
Velocity & 0.00603 & 0.00553 & 0.03454 & 0.02955 & 0.03537 \\
\hline
\end{tabular}

TABle 4: Time consumptions of the different algorithms for solving the $n$-DOF system in Example 3 (unit: second).

\begin{tabular}{lccccc}
\hline & CAA & & CQ-2x & & KR- $\alpha$ \\
& & $\rho_{\infty}=1$ & $\rho_{\infty}=0.8$ & $\rho_{\infty}=1$ & $\rho_{\infty}=0.8$ \\
\hline$n=200$ & 5.37478 & 1.63362 & 1.51627 & 3.75233 & 3.54338 \\
$n=500$ & 39.17923 & 14.80797 & 11.12231 & 31.67168 & 23.96452 \\
$n=1000$ & 246.31789 & 86.08239 & 91.89233 & 182.75176 & 183.34749 \\
$n=2000$ & 2045.64400 & 318.19379 & 492.85635 & 893.82694 & 1094.05923 \\
$n=4000$ & 14992.598 & 4811.754 & 6220.651 & 12205.040 & 14868.966 \\
\hline
\end{tabular}

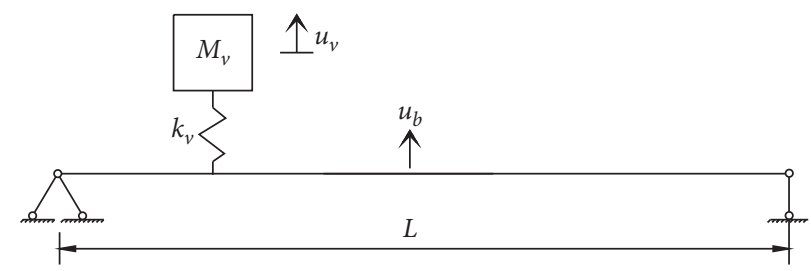

FIGURE 35: Beam with the moving spring mass. 


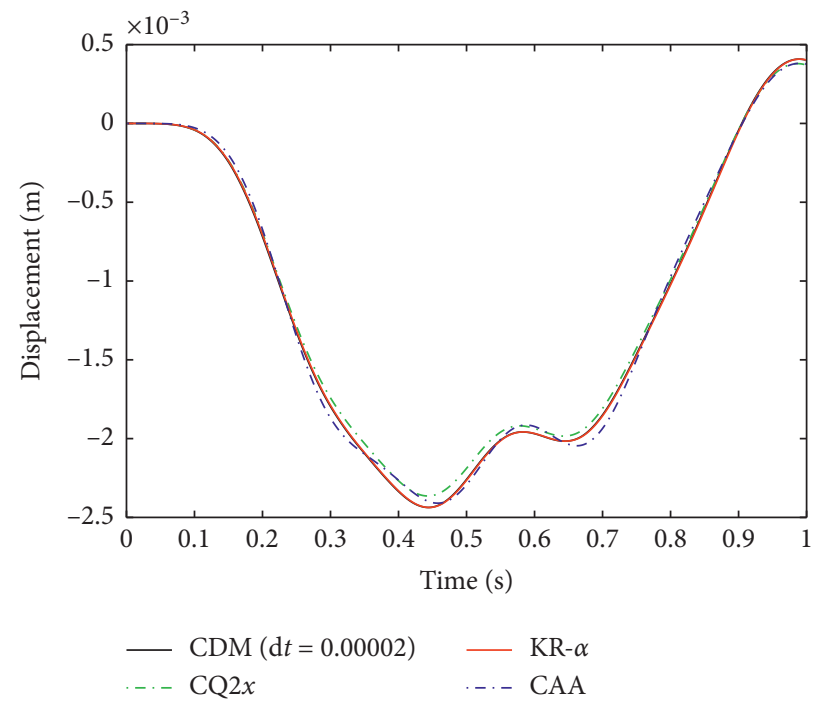

FIGURE 36: Deflection of the spring mass.

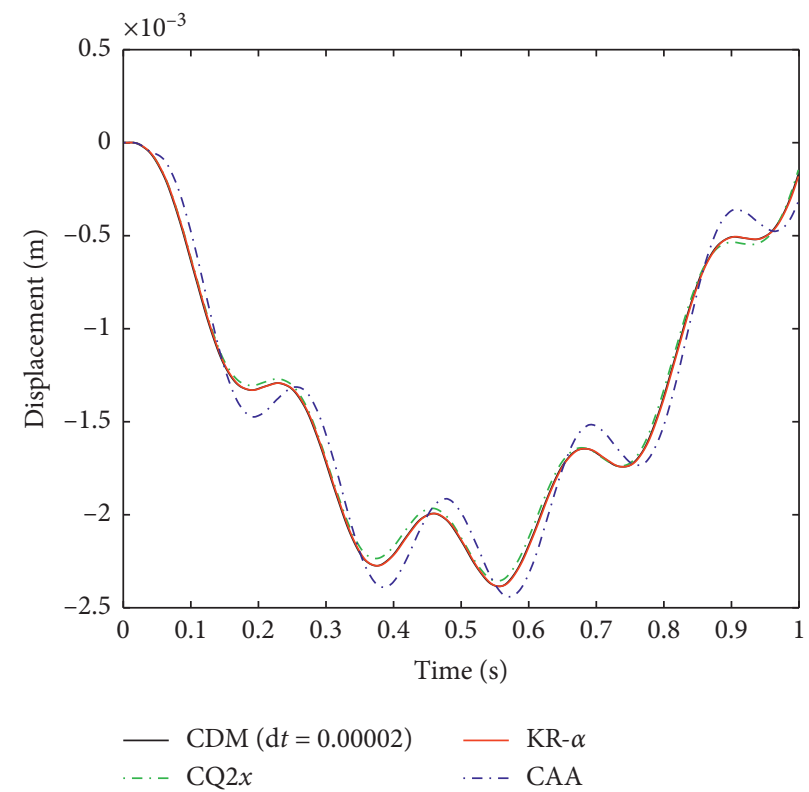

Figure 37: Midpoint vertical deflection of the beam.

TABle 5: Maximum deflection error ratios in Example 4.

\begin{tabular}{lccc}
\hline & CAA & CQ- $2 x\left(\rho_{\infty}=1\right)$ & KR- $\alpha\left(\rho_{\infty}=1\right)$ \\
\hline Midpoint of beam & 0.0672 & 0.0177 & 0.0061 \\
Spring mass & 0.0354 & 0.0303 & 0.0051 \\
\hline
\end{tabular}

TABLE 6: Time consumption of the different algorithms for solving the beam with the moving spring mass in Example 4 (unit: second).

\begin{tabular}{lccr}
\hline $\mathrm{CDM}$ & $\mathrm{CAA}$ & $\mathrm{CQ}-2 x\left(\rho_{\infty}=1\right)$ & $\mathrm{KR}-\alpha\left(\rho_{\infty}=1\right)$ \\
\hline 1153.89 & 1.40938 & 0.51252 & 1.21876 \\
\hline
\end{tabular}




\section{Conclusions}

A novel idea based on dimensional analysis for designing noniterative displacement algorithms to be applied in the analysis of nonlinear structural dynamics problems has been introduced and proven to be effective. Originating from only an objective function, a one-parameter family of two-step unconditionally stable noniterative displacement algorithms with controllable numerical energy dissipation has been developed. Analysis results show that the proposed CQ-2x method has unconditional stability in the cases of both stiffness hardening and softening. With the advantages of noniterative formulations of both displacement and velocity, controllable numerical dissipation, the use of one solver per time step, and no overshoot in either displacement or velocity, CQ- $2 x$ is expected to serve as a competitive displacement method for solving nonlinear structural dynamics problems.

\section{Appendix}

\section{A. Derivation of the Preliminary NDA Form}

When $u_{t+\Delta t}, m$, and $\Delta t$ are chosen as the basic dimensional units, equation (5) can be expressed in terms of dimensionless quantities as follows:

$$
\frac{u_{t+2 \Delta t}}{u_{t+\Delta t}}=\frac{u_{t+2 \Delta t}}{u_{t+\Delta t}}\left(1, \frac{u_{t}}{u_{t+\Delta t}}, \frac{f_{t+\Delta t} \Delta t^{2}}{m u_{t+\Delta t}}, 1, \frac{c_{t+\Delta t} \Delta t}{m}, \frac{k_{t+\Delta t} \Delta t^{2}}{m}, 1\right) .
$$

To construct the function $u_{t+2 \Delta t} / u_{t+\Delta t}$, a linear combination of $u_{t+2 \Delta t} / u_{t+\Delta t}, 1, u_{t} / u_{t+\Delta t}$, and $\left(f_{t+\Delta t} \Delta t^{2}\right) / m u_{t+\Delta t}$ can be used:

$$
r_{1} \frac{u_{t+2 \Delta t}}{u_{t+\Delta t}}=r_{2}+r_{3} \frac{u_{t}}{u_{t+\Delta t}}+r_{4} \frac{f_{t+\Delta t} \Delta t^{2}}{m u_{t+\Delta t}},
$$

where $r_{i}(i=1, \ldots, 4)$ are coefficients. The effects of the dimensionless arguments $c_{t+\Delta t} \Delta t / m$ and $k_{t+\Delta t} \Delta t^{2} / m$, which represent the structure's material properties, on the computed displacements can be expressed by replacing the coefficients $r_{i}(i=1,2,3)$ with linear combinations of $c_{t+\Delta t} \Delta t / m, k_{t+\Delta t} \Delta t^{2} / m$, and a constant term 1 as follows:

$$
\begin{aligned}
& r_{1}=l_{1}+l_{2} \frac{c_{t+\Delta t} \Delta t}{m}+l_{3} \frac{k_{t+\Delta t} \Delta t^{2}}{m} \\
& r_{2}=l_{4}+l_{5} \frac{c_{t+\Delta t} \Delta t}{m}+l_{6} \frac{k_{t+\Delta t} \Delta t^{2}}{m}, \\
& r_{3}=l_{7}+l_{8} \frac{c_{t+\Delta t} \Delta t}{m}+l_{9} \frac{k_{t+\Delta t} \Delta t^{2}}{m}
\end{aligned}
$$

where $l_{i}(i=1, \ldots, 9)$ are coefficients. Substituting equations (A.3a)-(A.3c) into equation (A.2) yields

$$
\begin{aligned}
\left(l_{1}+l_{2} \frac{c_{t+\Delta t} \Delta t}{m}+l_{3} \frac{k_{t+\Delta t} \Delta t^{2}}{m}\right) \frac{u_{t+2 \Delta t}}{u_{t+\Delta t}}= & \left(l_{4}+l_{5} \frac{c_{t+\Delta t} \Delta t}{m}+l_{6} \frac{k_{t+\Delta t} \Delta t^{2}}{m}\right) \\
& +\left(l_{7}+l_{8} \frac{c_{t+\Delta t} \Delta t}{m}+l_{9} \frac{k_{t+\Delta t} \Delta t^{2}}{m}\right) \frac{u_{t}}{u_{t+\Delta t}}+l_{10} \frac{f_{t+\Delta t} \Delta t^{2}}{m u_{t+\Delta t}}
\end{aligned}
$$

where $l_{10}=r_{4}$. Equation (A.4) can be simplified by multi-

plying by $m u_{t+\Delta t}$ as follows:

$$
\begin{aligned}
\left(l_{1} m+l_{2} c \Delta t+l_{3} k_{t+\Delta t} \Delta t^{2}\right) u_{t+2 \Delta t}= & \left(l_{4} m+l_{5} c \Delta t+l_{6} k_{t+\Delta t} \Delta t^{2}\right) u_{t+\Delta t} \\
& +\left(l_{7} m+l_{8} c \Delta t+l_{9} k_{t+\Delta t} \Delta t^{2}\right) u_{t}+l_{10} f_{t+\Delta t} \Delta t^{2}
\end{aligned}
$$

To reduce the number of undetermined coefficients $l_{i}$, equation (A.5) can be further simplified by dividing both sides by $l_{1}$ :

$$
\begin{aligned}
\left(m+b_{1} c \Delta t+b_{2} k_{t+\Delta t} \Delta t^{2}\right) u_{t+2 \Delta t}= & \left(b_{3} m+b_{4} c \Delta t+b_{5} k_{t+\Delta t} \Delta t^{2}\right) u_{t+\Delta t} \\
& +\left(b_{6} m+b_{7} c \Delta t+b_{8} k_{t+\Delta t} \Delta t^{2}\right) u_{t}+b_{9} f_{t+\Delta t} \Delta t^{2}
\end{aligned}
$$


where $\quad b_{i}=\left(l_{i+1} / l_{1}\right)(i=1, \ldots, 9)$ are undetermined coefficients.

B. Expressions for $e_{0}, e_{1}, e_{2}, e_{3}, e_{4}$, and $e_{5}$

Equation (A.6) is the preliminary NDA form of the CQ$2 x$ method.

$$
\begin{aligned}
& e_{0}=\left(1-b_{3}-b_{6}\right) m u_{(t)}, \\
& e_{1}=\left(2-b_{3}\right) m v_{(t)}+\left(b_{1}-b_{4}-b_{7}\right) c(t+\Delta t) u_{(t)}, \\
& e_{2}=\left(2-0.5 b_{3}-b_{9}\right) m a_{(t)}+\left(2 b_{1}-b_{4}-b_{9}\right) c(t+\Delta t) v_{(t)}+\left(b_{2}-b_{5}-b_{8}-b_{9}\right) k(t+\Delta t) u_{(t)}, \\
& e_{3}=\left(\frac{4}{3}-\frac{1}{6} b_{3}-b_{9}\right) m \dot{a}_{(t)}+\left(2 b_{1}-0.5 b_{4}-b_{9}\right) c(t+\Delta t) a_{(t)}+\left(2 b_{2}-b_{5}-b_{9}\right) k(t+\Delta t) v_{(t)}, \\
& e_{4}=\left(\frac{2}{3}-\frac{1}{24} b_{3}-\frac{1}{2} b_{9}\right) m \ddot{a}_{(t)}+\left(\frac{4}{3} b_{1}-\frac{1}{6} b_{4}-\frac{1}{2} b_{9}\right) c(t+\Delta t) \dot{a}_{(t)}+\left(2 b_{2}-\frac{1}{2} b_{5}-\frac{1}{2} b_{9}\right) k(t+\Delta t) a_{(t)}, \\
& e_{5}=\left(\frac{4}{15}-\frac{1}{120} b_{3}-\frac{1}{6} b_{9}\right) m \dot{t}_{(t)}+\left(\frac{2}{3} b_{1}-\frac{1}{24} b_{4}-\frac{1}{6} b_{9}\right) c(t+\Delta t) \ddot{a}_{(t)}+\left(\frac{4}{3} b_{2}-\frac{1}{6} b_{5}-\frac{1}{6} b_{9}\right) k(t+\Delta t) \dot{a}_{(t)} .
\end{aligned}
$$

The targets of computation may be either buildings or bridges, and the dynamic responses may be forced by wind or an earthquake; consequently, the values of $m, c(t+\Delta t)$, and $k(t+\Delta t)$ and the forms of $u_{(t)}, v_{(t)}, \dot{a}_{(t)}, \ddot{a}_{(t)}$, and $\stackrel{t}{a}_{(t)}$ in the algorithm are arbitrary. Thus, by referring to equations (B.1a)-(B.1f), the following can be derived:

$$
\begin{aligned}
& e_{0}=0 \longrightarrow 1-b_{3}-b_{6}=0, \\
& e_{1}=0 \longrightarrow 2-b_{3}=0, b_{1}-b_{4}-b_{7}=0, \\
& e_{2}=0 \longrightarrow 2-0.5 b_{3}-b_{9}=0 \text {, } \\
& 2 b_{1}-b_{4}-b_{9}=0 \text {, } \\
& b_{2}-b_{5}-b_{8}-b_{9}=0 \text {, } \\
& e_{3}=0 \longrightarrow \frac{4}{3}-\frac{1}{6} b_{3}-b_{9}=0 \text {, } \\
& 2 b_{1}-0.5 b_{4}-b_{9}=0 \text {, } \\
& 2 b_{2}-b_{5}-b_{9}=0 \text {, } \\
& e_{4}=0 \longrightarrow \frac{2}{3}-\frac{1}{24} b_{3}-\frac{1}{2} b_{9}=0 \text {, } \\
& \frac{4}{3} b_{1}-\frac{1}{6} b_{4}-\frac{1}{2} b_{9}=0 \text {, } \\
& 2 b_{2}-\frac{1}{2} b_{5}-\frac{1}{2} b_{9}=0 \text {, } \\
& e_{5}=0 \longrightarrow \frac{4}{15}-\frac{1}{120} b_{3}-\frac{1}{6} b_{9}=0 \text {, } \\
& \frac{2}{3} b_{1}-\frac{1}{24} b_{4}-\frac{1}{6} b_{9}=0 \text {, } \\
& \frac{4}{3} b_{2}-\frac{1}{6} b_{5}-\frac{1}{6} b_{9}=0 \text {. }
\end{aligned}
$$

\section{Derivation of the NDA Stability Requirements}

The characteristic equation of the amplification matrix $A$ is given by

$$
|A-\lambda I|=\lambda^{2}-2 A_{1} \lambda+A_{2}=0,
$$

where $\lambda$ is the root of the characteristic equation of $A$ and

$$
\begin{aligned}
& A_{1}=\frac{A_{11}+A_{22}}{2}, \\
& A_{2}=A_{11} A_{22}-A_{12} A_{21} .
\end{aligned}
$$

At the boundary line of the domain of NDA stability, the following holds:

$$
\rho(A)=1,
$$

where $\rho(A)$ is the spectral radius of the amplification matrix A.

Equation (C3) being satisfied means that

$$
|\lambda|_{\max }=1 \text {. }
$$

For a complex-valued $\lambda$, equation (C5) is satisfied only when

$$
\lambda=e^{i \varphi}
$$

where $i=\sqrt{-1}$ and $\varphi(\varphi \in[0,2 \pi])$ is the argument of $\lambda$.

Substituting equation (C5) into equation (C1) yields

$$
e^{i 2 \varphi}-2 A_{1} e^{i \varphi}+A_{2}=0
$$

Considering $e^{i \varphi}=\cos \varphi+i \sin \varphi, \cos 2 \varphi=2 \cos ^{2} \varphi-1$, and $\sin 2 \varphi=2 \sin \varphi \cos \varphi$, equation (C6) can be rewritten as 
$\left(2 \cos \varphi\left(\cos \varphi-A_{1}\right)+A_{2}-1\right)+i\left(2 \sin \varphi\left(\cos \varphi-A_{1}\right)\right)=0$.

Equation (C7) is satisfied only when

$$
\begin{array}{r}
2 \cos \varphi\left(\cos \varphi-A_{1}\right)+A_{2}-1=0, \\
2 \sin \varphi\left(\cos \varphi-A_{1}\right)=0 .
\end{array}
$$

The boundary line of the NDA stability domain can be determined from equations (C8a) and (C8b) when $\varphi \in[0,2 \pi]$. It can be derived that when $0<\varphi<\pi$ equations (C8a) and (C8b)are satisfied when

$$
\begin{aligned}
& A_{1}=\cos \varphi, \\
& A_{2}=1 .
\end{aligned}
$$

When $\varphi=0$ and $\varphi=2 \pi$, equations (C8a) and (C8b) are satisfied when

$$
1-2 A_{1}+A_{2}=0
$$

When $\varphi=\pi$, equations $(\mathrm{C} 8 \mathrm{a})$ and $(\mathrm{C} 8 \mathrm{~b})$ are satisfied when

$$
1+2 A_{1}+A_{2}=0 \text {. }
$$

The boundary line of the NDA stability domain is thus composed of three lines described by equations (C9), (C10), and $(\mathrm{C} 11)$ in the $A_{1}-A_{2}$ plane, as shown in Figure 1. Because $\rho(A)$ is a continuous function of $A_{1}$ and $A_{2}$, when $A_{1}=A_{2}=0$ and $\rho(A)=0<1$, the domain of NDA stability is the inner part of the triangle shown in Figure 1 and can be expressed as

$$
\begin{gathered}
\%-1 \leq A_{2} \leq 1, \\
-\frac{1}{2}\left(1+A_{2}\right) \leq A_{1} \leq \frac{1}{2}\left(1+A_{2}\right) .
\end{gathered}
$$

\section{Dissipation and Dispersion}

The characteristic values of $\lambda$ in equation (C1) are

$$
\begin{aligned}
& \lambda_{1,2}=A_{1} \pm \sqrt{A_{2}-A_{1}^{2}} i, \quad \text { when } A_{2} \geq A_{1}^{2}, \\
& \lambda_{1,2}=A_{1} \pm \sqrt{A_{1}^{2}-A_{2}}, \quad \text { when } A_{2} \leq A_{1}^{2} .
\end{aligned}
$$

The spectral radius of the amplification matrix $A$ is

$$
\begin{aligned}
& \% \rho(A)=\sqrt{A_{2}}, \quad \text { when } A_{2} \geq A_{1}^{2}, \\
& \rho(A)=\max \left|A_{1} \pm \sqrt{A_{1}^{2}-A_{2}}\right|, \quad \text { when } A_{2} \leq A_{1}^{2} .
\end{aligned}
$$

When $A_{2} \geq A_{1}^{2}$, equation (D1a) can be rewritten as [18] $\lambda_{1,2}=\sigma \pm \varepsilon i=A_{1} \pm \sqrt{A_{2}-A_{1}^{2}} i=\exp \left[\bar{\Omega}\left(-\bar{\xi} \pm \sqrt{1-\bar{\xi}^{2}} i\right)\right]$ where the numerical circular frequency $\bar{\Omega}$ is given by

$$
\bar{\Omega}=\bar{\omega}_{n} \Delta t=\frac{\tan ^{-1}(\varepsilon / \sigma)}{\sqrt{1-\bar{\xi}^{2}}}=\frac{\tan ^{-1}\left(\sqrt{A_{2}-A_{1}^{2}} / A_{1}\right)}{\sqrt{1-\bar{\xi}^{2}}},
$$

and the numerical damping ratio $\bar{\xi}$ can be written as

$$
\bar{\xi}=-\frac{\ln \left(\sigma^{2}+\varepsilon^{2}\right)}{2 \bar{\Omega}}=-\frac{\ln \left(A_{2}\right)}{2 \bar{\Omega}} \text {. }
$$

The relative period error $\mathrm{Pe}$ is written as

$$
\mathrm{Pe}=\frac{\Omega}{\bar{\Omega}}-1 \text {. }
$$

\section{Data Availability}

All data included in this study are available from the first author lcq_stu@126.com upon request.

\section{Conflicts of Interest}

The authors declare that they have no conflicts of interest.

\section{Acknowledgments}

This research was jointly supported by the National Natural Science Foundation of China (Grant no. U1934207) and Innovation-Driven Project of Central South University (Grant no. 502501006).

\section{References}

[1] T. R. Hughes, The Finite Element Method Linear Static and Dynamic Finite Element Analysis, Prentice-Hall, Upper Saddle River, NJ, USA, 2000.

[2] K.-J. Bathe, Finite Element Procedures, Prentice-Hall, Upper Saddle River, NJ, USA, 2014.

[3] C. Houbolt John, "A recurrence matrix solution for the dynamic response of aircraft in gusts," Journal of Aeronautical Science, vol. 17, no. 1950, pp. 540-550, 1950.

[4] N. M. Newmark, "A method of computation for structural dynamics," Journal of the Engineering Mechanics Division, vol. 85, no. 1, pp. 67-94, 1959.

[5] L. Wilson, A Computer Program for the Dynamic Stress Analysis of Underground Structure, SESM Univ.California, Berkeley, CA, USA, 1968.

[6] H. M. Hilber, T. J. R. Hughes, and R. L. Taylor, "Improved numerical dissipation for time integration algorithms in structural dynamics," Earthquake Engineering \& Structural Dynamics, vol. 5, no. 3, pp. 283-292, 1977.

[7] W. L. Wood, M. Bossak, and O. C. Zienkiewicz, "An alpha modification of Newmark's method," International Journal for Numerical Methods in Engineering, vol. 15, no. 10, pp. 1562-1566, 1980.

[8] J. Chung and G. M. Hulbert, "A time integration algorithm for structural dynamics with improved numerical dissipation: the generalized- $\alpha$ method," Journal of Applied Mechanics, vol. 60, no. 2, pp. 371-375, 1993.

[9] B. Klaus-Jrgen and B. M. M. Irfan, "On a composite implicit time integration procedure for nonlinear dynamics," Computers and Structures, vol. 83, no. 31-32, pp. 2513-2524, 2005. 
[10] B. Klaus Juergen, "Conserving energy and momentum in nonlinear dynamics A simple implicit time integration scheme," Computers and Structures, vol. 85, no. 7-8, pp. 437-445, 2007.

[11] K.-J. Bathe, G. Noh, and G. Noh, "Insight into an implicit time integration scheme for structural dynamics," Computers \& Structures, vol. 98, no. 5, pp. 1-6, 2012.

[12] G. M. Hulbert and J. Chung, "Explicit time integration algorithms for structural dynamics with optimal numerical dissipation," Computer Methods in Applied Mechanics and Engineering, vol. 137, no. 2, pp. 175-188, 1996.

[13] S.-Y. Chang and Yih, "Explicit pseudodynamic algorithm with unconditional stability," Journal of Engineering Mechanics, vol. 128, no. 9, pp. 935-947, 2002.

[14] S. Chang and Yih, "An explicit method with improved stability property," International Journal for Numerical Methods in Engineering, vol. 77, no. 8, pp. 1100-1120, 2010.

[15] S. Chang and Yih, "Family of structure-dependent explicit methods for structural dynamics," Journal of Engineering Mechanics, vol. 140, no. 6, Article ID 06014005, 2014.

[16] S.-Y. Chang, "Dissipative, noniterative integration algorithms with unconditional stability for mildly nonlinear structural dynamic problems," Nonlinear Dynamics, vol. 79, no. 2, pp. 1625-1649, 2015.

[17] C. Chen and J. M. Ricles, "Development of direct integration algorithms for structural dynamics using discrete control theory," Journal of Engineering Mechanics, vol. 134, no. 8, pp. 676-683, 2008

[18] K. Chinmoy and M. Ricles James, "Development of a family of unconditionally stable explicit direct integration algorithms with controllable numerical energy dissipation," Earthquake Engineering and Structural Dynamics, vol. 43, no. 43, pp. 1361-1380, 2014.

[19] K. Chinmoy and M. Ricles James, "Assessment of explicit and semi explicit classes of model based algorithms for direct integration in structural dynamics," International Journal for Numerical Methods in Engineering, vol. 107, no. 1, pp. 49-73, 2016.

[20] H. Jason and K. Tamma Kumar, Advanced Computational Dynamics of Particles, Materials, and Structures, John Wiley, New York, NY, USA, 2012.

[21] K. K. Tamma, J. Har, X. Zhou, M. Shimada, and A. Hoitink, "An overview and recent advances in vector and scalar formalisms: space/time discretizations in computational dynamics-A unified approach," Archives of Computational Methods in Engineering, vol. 18, no. 2, pp. 119-283, 2011.

[22] C. Li, J. Junping, L. Jiang, and T. Yang, "Theory and implementation of a two-step unconditionally stable explicit integration algorithm for vibration analysis of structures," Shock and Vibration, vol. 2016, Article ID 2831206, 10 pages, 2016.

[23] C.Q. Li and L. Jiang, "Unconditionally stable explicit displacement method for analyzing nonlinear structural dynamics problems," Journal of Engineering Mechanics(ASCE), vol. 144, no. 9, Article ID 04018079, 2018.

[24] C.1ing $\mathrm{Li}$ and M. Lou, "Central-eccentric method(In Chinese)," Journal of Tongji University(for Natural Science), vol. 39, no. 2, pp. 179-186, 2011.

[25] H. M. Hilber and T. J. R. Hughes, "Collocation, dissipation and [overshoot] for time integration schemes in structural dynamics," Earthquake Engineering \& Structural Dynamics, vol. 6, no. 1, pp. 99-117, 1978.
[26] S. Aram and F. Jamshid, "A unified starting procedure for the Houbolt method," Communications in Numerical Methods in Engineering, vol. 24, no. 1, pp. 1-13, 2010.

[27] V. A. Leontyev, "Direct time integration algorithm with controllable numerical dissipation for structural dynamics: two-step Lambda method," Applied Numerical Mathematics, vol. 60, no. 3, pp. 277-292, 2010.

[28] B. E. Hairer and G. Wanner, Solving Ordinary Differential Equations II Stiff and Differential-Algebraic Problems, Prentice-Hall, Upper Saddle River, NJ, USA, 1996.

[29] Y. M. Xie, "An assessment of time integration schemes for non-linear dynamic equations," Journal of Sound and Vibration, vol. 192, no. 1, pp. 321-331, 1996.

[30] W. L. Wood and M. E. Oduor, "Stability properties of some algorithms for the solution of nonlinear dynamic vibration equations," Communications in Applied Numerical Methods, vol. 4, no. 2, pp. 205-212, 1988.

[31] S.-Y. Chang, "Improved explicit method for structural dynamics," Journal of Engineering Mechanics, vol. 133, no. 7, pp. 748-760, 2007.

[32] Y. B. Yang, J. D. Yau, and W. S. Wu, Vehicle-Bridge Interaction Dynamics with Applications to High-Speed Railways, World Scientific Publishing Co.Pte.Ltd, Singapore, 2004. 